OPEN ACCESS

Edited by:

Babatunde Ogunnaike, University of Delaware, United States

Reviewed by:

Filippo Tempia

University of Turin, Italy

Ilya Bezprozvanny,

UT Southwestern Medical Center,

United States

*Correspondence:

Naveed Aslam

naveed.aslam@biosystomics.com

Specialty section:

This article was submitted to

Systems Biology,

a section of the journa

Frontiers in Neuroscience

Received: 02 August 2019 Accepted: 11 December 2019

Published: 31 January 2020

Citation:

Aslam N and Alvi F (2020) Simplified Model of PKC $\gamma$ Signaling

Dysregulation and

Cytosol-to-Membrane Translocation Kinetics During Neurodegenerative

Spinocerebellar Ataxia Type 14 (SCA14). Front. Neurosci. 13:1397.

doi: 10.3389/fnins.2019.01397

\section{Simplified Model of PKC $\gamma$ Signaling Dysregulation and Cytosol-to-Membrane Translocation Kinetics During Neurodegenerative Spinocerebellar Ataxia Type 14 (SCA14)}

\author{
Naveed Aslam ${ }^{1,2 *}$ and Farah Alvi ${ }^{1,3}$ \\ ${ }^{1}$ BioSystOmics, Bellaire, TX, United States, ${ }^{2}$ Department of Chemistry and Chemical Engineering, Lahore University \\ of Management Sciences, Lahore, Pakistan, ${ }^{3}$ Department of Physics, COMSATS University Islamabad, Islamabad, Pakistan
}

Spinocerebellar ataxia type 14 (SCA14) is an autosomal neurodegenerative disease clinically characterized by progressive ataxia in the patient's gait, accompanied by slurred speech and abnormal eye movements. These symptoms are linked to the loss of Purkinje cells (PCs), which leads to cerebellar neurodegeneration. PC observations link the mutations in PRKCG gene encoding protein kinase $C \gamma(P K C \gamma)$ to SCA14. Observations also show that the link between PKC $\gamma$ and SCA14 relies on a gainof-function mechanism, and, in fact, both positive and negative regulation of PKC $\gamma$ expression and activity may result in changes in cellular number, size, and complexity of the dendritic arbors in PCs. Here, through a systems biology approach, we investigate a key question relating to this system: why is $\mathrm{PKC} \gamma$ membrane residence time reduced in SCA14 mutant PCs compared to wild-type (WT) PCs? In this study, we investigate this question through two contrasting PKC $\gamma$ signaling models in PCs. The first model proposed in this study describes the mechanism through which $\mathrm{PKC} \gamma$ signaling activity may be regulated in WT PCs. In contrast, the second model explores how mutations in PKC $\gamma$ signaling affect the state of SCA14 in PCs. Numerical simulations of both models show that, in response to extracellular stimuli-induced depolarization of the membrane compartment, PKC $\gamma$ and diacylglycerol kinase $\gamma(D G K \gamma)$ translocate to the membrane. Results from our computational approach indicate that, for the same set of parameters, PKC $\gamma$ membrane residence time is shorter in the SCA14 mutant model compared to the WT model. These results show how PKC $\gamma$ membrane residence time is regulated by diacylglycerol (DAG), causing translocated PKC $\gamma$ to return to the cytosol as DAG levels drop. This study shows that, when the strength of the extracellular signal is held 
constant, the membrane lifetime of mutant PKC $\gamma$ is reduced. This reduction is due to the presence of constitutively active mutant PKC $\gamma$ in the cytosol. Cytosolic PKC $\gamma$, in turn, leads to phosphorylation and activation of DGK $\gamma$ while it is still residing in the cytosol. This effect occurs even during the resting conditions. Thus, the SCA14 mutant model explains that, when both DAG effector molecules are active in the cytosol, their interactions in the membrane compartment are reduced, critically influencing PKC $\gamma$ membrane residence time.

Keywords: PKC $\gamma$ translocation kinetics, dysregulated signaling, spinocerebellar ataxia, neurodegeneration, mutant

\section{INTRODUCTION}

Clinically, the term "ataxia" describes abnormal limb movements and poor limb coordination (Shimobayashi, 2016). The most common form of ataxia is cerebellar ataxia, which can be linked to dysfunction either within the cerebellum or the cerebellar connecting pathways (Shimobayashi, 2016). Spinocerebellar ataxia (SCA) is a disease that manifests as dysfunction in the spinocerebellum, the part of the cerebellar cortex that receives somatosensory input from the spinal cord (Soong and Paulson, 2007; Carlson et al., 2009; Paulson, 2009). SCA14 is a rare form of SCA that can be inherited through autosomal dominance (Duenas et al., 2006; Shimobayashi, 2016). SCA14 has been linked to missense point mutations, deletions, or splice site mutations in the PRKCG coding region of PKC $\gamma$ (Yamashita et al., 2000). Previous studies have linked almost 30 types of deletions or missense mutations in the PRKCG gene to SCA14-related symptoms (Shimobayashi, 2016). PKC $\gamma$ is principally expressed in the central nervous system (CNS) and predominantly found in PCs (Saito et al., 1988; Saito and Shirai, 2002; Schrenk et al., 2002; Yabe et al., 2003). PKC $\gamma$ is considered one of the key factors that control cerebellar development. SCA14 disease onset ranges from childhood to late adulthood, and usually does not result in a shorter lifespan. Generally, clinical symptoms of SCA14 include ataxia, dysarthria, oculomotor dysfunction, vertigo, facial myokymia, and myoclonus (Shimobayashi, 2016). Post-mortem neurohistological-pathological studies of patients with SCA14 have shown a pronounced reduction in the number of cerebellar Purkinje cells (PCs), as well a reduction in cellular size and complexity of the dendritic arbor (Brkanac et al., 2002).

An interesting possibility, supported by previous experimental observations, is that SCA14 might be linked to increases in PKC $\gamma$ activity (Metzger and Kapfhammer, 2000; Adachi et al., 2008; Shimobayashi, 2016; Wong et al., 2018). Studies have shown that PMA-induced chronic PKC $\gamma$ activation in cerebellar slice cultures drastically inhibits the growth and development of the PC dendritic tree (Metzger and Kapfhammer, 2000). This result could indicate that degeneration of the PC dendritic tree during SCA14 may be caused by increased PKC $\gamma$ activity (Verbeek et al., 2005). Furthermore, 19 out of 20 spontaneous mutations found in the PKC $\gamma$ gene of SCA14 patients showed increased constitutive PKC $\gamma$ activity (Adachi et al., 2008). How constitutively active $\mathrm{PKC} \gamma$ may contribute to neurodegeneration is not clear. However, there is evidence that, despite an enhanced basal activity of the PKC $\gamma$ isoform, there may be a deficit recruitment or regulation of downstream targets linked to a loss of specific cerebellar functions.

One possibility may be related to the membrane residence duration of PKC $\gamma$ (Adachi et al., 2008; Shuvaev et al., 2011; Wong et al., 2018). In order to achieve precise regulation of PKC $\gamma s^{\prime}$ downstream targets, its membrane residence time must be exquisitely regulated (Adachi et al., 2008; Shuvaev et al., 2011; Shimobayashi, 2016). If the amount of time PKC $\gamma$ spends in the membrane compartment is altered, this may lead to aberrant regulation and/or recruitment of downstream signaling molecules. Previous observations suggest that in PCs, the PKC $\gamma$ membrane residence time is controlled by the amplitude and duration of DAG signaling. Experimental observation in Chinese hamster ovary $(\mathrm{CHO})$ cells indicate that DAG signaling could contribute to a functional coupling of $\mathrm{PKC} \gamma$ and its regulator DGK $\gamma$ (Yamaguchi et al., 2006; Goto and Kondo, 1999; Mérida et al., 2008). These results show (Yamaguchi et al., 2006) that in the membrane compartment, DGK $\gamma$ regulates the activity of PKC $\gamma$ through phosphorylating DAG, and thus inducing its metabolism. Our previous work elucidated the role of this functional coupling and showed how the timing of DGK $\gamma$ and PKC $\gamma$ colocalization in the membrane compartment is important for DAG signaling regulation in both $\mathrm{CHO}$ cells and PCs (Aslam and Alvi, 2017, 2019).

In addition, previous data indicate that, compared to wildtype, mutant PKC $\gamma$ has higher basal level activity, but reduced membrane compartment residence time (Shuvaev et al., 2011). This may explain aberrant downstream signaling in mutant models of PKC $\gamma$. For example, one study revealed a decrease in $\mathrm{PKC} \gamma$ signaling, measured by canonical transient receptor potential channel (TRPC3) phosphorylation, when a PKC $\gamma$ mutant remained at the membrane for significantly less time than wild-type PKC $\gamma$ (Adachi et al., 2008). Altered PKC $\gamma$ phosphorylation or recruitment may contribute to cerebellar dysfunction and apoptosis in PCs.

The purinergic receptors belong to a class of plasma membrane molecules that have been critically implicated in the regulation of physiological and pathological responses such as learning memory, inflammation, motor control, and sleep. They are classified into $\mathrm{P}_{1}$ (adenosine-activated) and $\mathrm{P}_{2}$ (ATP-activated) subfamilies. Among the $\mathrm{P}_{2}$ subfamily, the $\mathrm{P}_{2} \mathrm{X}$ subgroup is ionotropic and the $\mathrm{P}_{2} \mathrm{Y}$ subgroup is metabotropic nucleotide receptor. The $\mathrm{P}_{2} \mathrm{Y}$ subfamily has 
eight members and are expressed in cells of the nervous system. Among this subgroup, $\mathrm{P}_{2} \mathrm{Y}_{1}, \mathrm{P}_{2} \mathrm{Y}_{2}, \mathrm{P}_{2} \mathrm{Y}_{4}, \mathrm{P}_{2} \mathrm{Y}_{6}$, and $\mathrm{P}_{2} \mathrm{Y}_{11}$ receptors are coupled to $\mathrm{G}_{\mathrm{q}}$-protein (Weisman et al., 2012; Guzman and Gerevich, 2016). The activation of this group by general currency in energy conversion, i.e., adenosine triphosphate (ATP) or nucleotide, uridine-5'triphosphate (UTP), induces the $\mathrm{G}_{\mathrm{q}}$-dependent activation of phospholipase C (PLC), which promotes the hydrolysis of plasma membrane phospholipid phosphatidylinositol 4,5, bisphosphate $\left(\mathrm{PIP}_{2}\right)$ to generate second messenger DAG and inositol 1,4,5 triphosphate $\left(\mathrm{IP}_{3}\right)$. Both these second messengers are critical for the release of intracellular $\mathrm{Ca}^{+2}$ from stores, exchange of $\mathrm{Ca}^{+2}$ with extracellular pools, and activation of $\mathrm{PKC} \gamma$ and DGK $\gamma$.

This work explores why $\mathrm{PKC} \gamma$ membrane residence time is reduced in PCs in the mutant model, despite higher kinase activity. The following experimental observations provide the basis for this study: (1) Depolarization-induced activation of mGluR1 pathways leads to the membrane translocation of both mutant and wild-type PKC $\gamma$ (Shuvaev et al., 2011; Shimobayashi, 2016). (2) Mutant PKC $\gamma$ is constitutively active (Adachi et al., 2008; Shuvaev et al., 2011; Shimobayashi, 2016). (3) During a depolarization-induced activation event, the wildtype PKC $\gamma$ membrane residence time is $19 \mathrm{~s}$. The mutant PKC $\gamma$ membrane residence time is $6 \mathrm{~s}$ (Shuvaev et al., 2011). (4) Depolarization-induced stimulation of both mutant and wildtype $\mathrm{PKC} \gamma$ results in rapid membrane translocation followed by a slow return to the cytosol (Adachi et al., 2008; Shuvaev et al., 2011; Shimobayashi, 2016). (5) In response to ATP stimulation, $\mathrm{PKC} \gamma$ and DGK $\gamma$ form a subtype-specific functional coupling, which regulates DAG signaling in the membrane compartment in the $\mathrm{CHO}$ cell system (Yamaguchi et al., 2006). (6) Observations in post-mortem SCA14 cerebellum and human patient-derived induced pluripotent stem cells (iPSCs) show that, when activated, wild-type PKC $\gamma$ is distributed in the membrane and cytosolic compartment, whereas mutant PKC $\gamma$ tends to localize in cytosol (Wong et al., 2018), thus indicating that mutant $\mathrm{PKC} \gamma$ is impaired in its ability to translocate, or be retained at, the plasma membrane. (7) Additional observations from SCA14 iPSCs and SCA14 PCs indicate that mutant $\mathrm{PKC} \gamma$ present in cytosol is in hyperactive state (Wong et al., 2018). (8) Moreover, this molecule leads to a robust increase in $\mathrm{PKC} \gamma$ substrate phosphorylations as observed by increase in $\mathrm{PKC} \gamma$ substrate antibody and its well-established target, i.e., myristoylated alanine-rich C-kinase substrate (MARCKS) in SCA14 cerebellum (Wong et al., 2018). In this work, we test the hypothesis that due to reduced local signaling, the lifetime of mutant PKC $\gamma$ is reduced in the membrane compartment.

This work tests this hypothesis by constructing two contrasting models (WT and mutant). The simulations mimicking mutant model includes a constitutively active PKC $\gamma$ in the cytosol (Adachi et al., 2008; Shuvaev et al., 2011; Shimobayashi, 2016; Wong et al., 2018), which, in turn, phosphorylates and activates DGK $\gamma$ even during basal conditions. The simulations representing the wild-type model includes inactive and dormant $\mathrm{PKC} \gamma$ and DGK $\gamma$ in the cytosol during basal conditions and models translocation to the membrane compartment upon stimulation. The wild-type model shows membrane activation of DGK $\gamma$ in a stimulation-dependent manner, whereas the mutant model relies on stimulation-independent cytosolic DGK $\gamma$ activation. Both models are numerically perturbed by the same stimulation strength levels. For both models, all kinetic rate constants and translocation parameters are set at the identical numerical values. The overall goal of this study is to compare the effects on PKC $\gamma$ membrane residence duration in the mutant and wild-type models through numerical experimentation, with all other parameters held constant. Here, we show that, despite higher activity levels membrane residence, duration for $\mathrm{PKC} \gamma$ in the mutant model is three times shorter than the wild-type. We also show that, when a stimulation pulse is applied to mimic depolarization-induced activation of mGluR1, PKC $\gamma$ translocation from the cytosol to the membrane is induced. The manner and time scale of this induction is consistent with experimental observations (Shuvaev et al., 2011). Depolarization-induced local DAG generation in the membrane compartment also induces DGK $\gamma$ translocation from the cytosol to the membrane, where the molecule acts as a key negative regulator of DAG levels in the membrane compartment.

Experimental results suggest that despite the constitutively active nature of mutant molecule, the impaired translocation and shorter membrane residence time result in lower $\mathrm{PKC} \gamma$ concentrations in the membrane compartment of PCs (Shuvaev et al., 2011; Wong et al., 2018). This observation could be linked to the association of mutant $\mathrm{PKC} \gamma$ with large EPSC amplitudes in PCs (Shuvaev et al., 2011). Our simulations are consistent with these observations, as the maximum membrane-to-cytosol (M/C) ratio in the mutant model is half of the ratio in the wild-type model. This indicates that reductions in PKC $\gamma$ concentration in the membrane compartment may be linked to impaired PC functionality.

\section{MATERIALS AND METHODS}

\section{Biochemical Reactions}

The following model describes a mutant PKC $\gamma$ signaling model in PCs. This simulation models mutant and active PKC $\gamma$ and DGK $\gamma$ molecules. These biochemical interactions describe how local DAG generation leads to a signaling loop between PKC $\gamma$ and DGK $\gamma$ in our mutant models of PCs. The simulated interactions of molecules within the PKC $\gamma$ and $\mathrm{DKC} \gamma$ loop (Figure 1) are based on standard Michaelis-Menten kinetics. The following sets of biochemical reactions are used to describe the molecular interactions that occur within the PKC $\gamma$ and DKC $\gamma$ loop. The dynamic variables are DAG, DGK $\gamma$, and $\mathrm{PKC} \gamma$. Subscript I represents the concentration in the first compartment, the plasma membrane. Subscript II denotes the concentration in the second compartment, which represents the cytosol. The superscript Active represents the activated form of a molecule and the subscript $\mathrm{P}$ represents the phosphorylated 


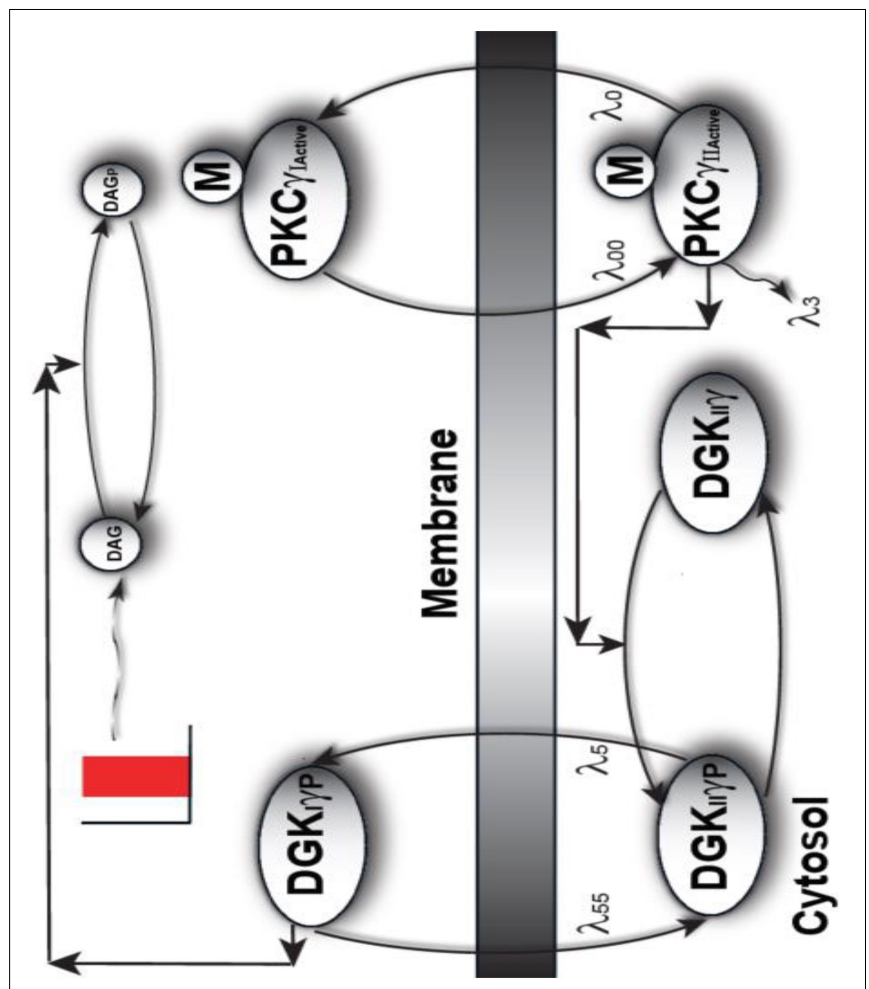

FIGURE 1 | A proposed regulatory model of mutant PKC $\gamma$, translocation in the SCA14-associated Purkinje cells of the cerebellum. This is a two-compartment model where one compartment is cytosol, whereas the other compartment is plasma membrane. This model provides the mechanistic basis of how the translocation of mutant PKC $\gamma$ and DGK $\gamma$ molecules could be regulated in SCA14 disease. This model suggests that in diseases associated with $\mathrm{CPCs}$, the mutant $\mathrm{PKC} \gamma$ is constitutively active and resides in the cytosol. In turn, this constitutively active molecule induces the phosphorylation and activation of the cytosolic DGK $\gamma$ molecule even during the basal or unstimulated conditions. In contrast, to the wild-type model, the current model suggests that both PKC $\gamma$ and DGK $\gamma$ are active and cytosolic even during basal conditions. Depolarization-induced activation of purinergic receptor leads to the local generation of DAG and, in turn, induces the translocation of both mutant PKC $\gamma$ and DGK $\gamma$ from cytosol to membrane. Once in the plasma membrane compartment, the already active DGK $\gamma$ molecule directly converts DAG to PA through DAG phosphorylation.

form of a molecule. The subscript Mutant represents the mutant form of a molecule. The phosphatase $\mathrm{P}$ is approximated as a fixed parameter. The parameter S1 denotes purinergic receptor stimulation, which leads to the rapid generation of DAG molecules.

$$
\begin{gathered}
\text { PKC }^{\text {Active }} \gamma_{\text {II.Mutant }} \stackrel{\lambda_{0}}{\longrightarrow} \text { PKC }^{\text {Active }} \gamma_{\text {IMutant }} \\
\text { PKC }^{\text {Active }} \gamma_{\text {IMutant }} \stackrel{\lambda_{00}}{\longrightarrow} \text { PKC }^{\text {Active }} \gamma_{\text {II Mutant }} \\
\text { DGK } \gamma_{\text {IIP }} \stackrel{\lambda_{5}}{\longrightarrow} \text { DGK } \gamma_{1 P} \\
\text { DGK } \gamma_{\text {IP }} \stackrel{\lambda_{55}}{\longrightarrow} \text { DGK } \gamma_{\text {IIP }} \\
\mathrm{S}_{1} \stackrel{k_{1}}{\longrightarrow} \text { DAG }
\end{gathered}
$$

$$
\begin{aligned}
& \text { DGK } \gamma_{\text {II }}+\text { PKC }^{\text {Active }} \gamma_{\text {IIMutant }} \\
& \stackrel{\stackrel{\mathrm{k}_{2}}{\longrightarrow}}{\underset{\mathrm{k}_{3}}{\longrightarrow}} \mathrm{C}_{1} \stackrel{\mathrm{k}_{4}}{\longrightarrow} \mathrm{DGK} \gamma_{\mathrm{IP}}+\mathrm{PKC}^{\text {Active }} \gamma_{\text {IIMutant }} \\
& \mathrm{DGK} \gamma_{\mathrm{IIp}}+\mathrm{P} \stackrel{\mathrm{k}_{5}}{\longrightarrow} \mathrm{DGK} \gamma_{\mathrm{II}}+\mathrm{P} \\
& \mathrm{DAG}+\mathrm{DGK} \gamma_{\mathrm{IP}} \stackrel{\mathrm{k}_{6}}{\underset{\mathrm{k}_{7}}{\longrightarrow}} \mathrm{C}_{2} \stackrel{\mathrm{k}_{8}}{\longrightarrow} \mathrm{DGK} \gamma_{\mathrm{IP}}+\mathrm{DAG}_{\mathrm{P}} \\
& \text { DAGP }_{\mathrm{P}} \stackrel{\mathrm{kg}_{\mathrm{g}}}{\longrightarrow} \text { P.A } \\
& \mathrm{PKC}_{\mathrm{II}} \gamma^{\text {Active }} \text { mutant } \stackrel{\lambda 3}{\longrightarrow}[]
\end{aligned}
$$

The signaling described in the above model starts during basal conditions. The constitutively active cytosolic mutant PKC $\gamma$ molecule leads to phosphorylation and activation of DGK $\gamma$ in the cytosol. This event is described by Equation (6). A pulse stimulation that mimics depolarization-induced stimulation of the pathway leads to DAG generation at the plasma membrane. This local event, causing generation of a second messenger, is described in Equation (5). DAG generation then stimulates the migration of dormant and active mutant $\mathrm{PKC} \gamma_{\mathrm{II}}$ and $\mathrm{DGK} \gamma_{\mathrm{II}}$ from the cytosol to the plasma membrane. These migration events are described by Equations (1) and (3). Here, the migration rates " $\lambda_{0}$ " and " $\lambda_{5}$ " are described through functions that are directly proportional to DAG concentrations, but with different slopes. Then, the active $\gamma$-molecules in the plasma membrane compartment re-translocate to the cytosol with fixed migration rates, " $\lambda_{00}$ " and " $\lambda_{55}$ ", as described by Equations (2) and (4). Once at the plasma membrane, DGK $\gamma_{\text {I }}$ causes DAG metabolism through its phosphorylation, as described by Equation (8). Phosphorylated DAG is converted to phosphatidic acid (PA) as shown in Equation (9). PA is another key signaling lipid that may function directly as a key regulatory molecule. The dephosphorylation event of DGKII $\gamma_{\mathrm{P}}$ is described by Equation (7).

\section{Induction}

During simulations, the membrane depolarization-induced activation of purinergic receptor is mimicked through the application of a brief pulse. The local biosynthesis of second messenger DAG is modulated through a 1.0-min pulse stimulation. DAG generation in the membrane compartment induces the translocation and activation of its effector molecules.

\section{Temporal Dynamics}

The differential equations resulting from the above interactions [Equations (1) to (9)] were integrated through nonlinear solvers using MATLAB (MathWorks). The dynamical coefficients' values were estimated from limited experimental data (Shuvaev et al., 2011). Unknown rate constants were scaled to obtain dynamics that were comparable to experimental values (Shuvaev et al., 2011). Unless otherwise stated, all of the molecular concentrations in the model are expressed as $\mathrm{pg} / \mathrm{ml}$ and time is represented in seconds. 


\section{RESULTS}

\section{Comparative Models Describing DAG Signaling in Mutant and Wild-Type PCs}

The DAG signaling model we propose for mutant PCs (Figure 1) in cerebellar PCs is composed of two molecular components. The first component is mutant $\mathrm{PKC} \gamma$, which can be active and cytosolic (PKC ${ }^{\text {Active }} \gamma_{\text {II-Mutant }}$ ) or active and membrane (PKC $\left.{ }^{\text {Active }} \gamma_{\mathrm{I}-\text { Mutant }}\right)$. The second component is DGK $\gamma$, which can be cytosolic and inactive (DGK $\left.\mathrm{DI}_{\mathrm{II}} \gamma\right)$; cytosolic, active, and phosphorylated $\left(\mathrm{DGK}_{\mathrm{II}} \gamma_{\mathrm{P}}^{\mathrm{A}}\right)$; or active and phosphorylated in the membrane compartment $\left(\mathrm{DGK}_{\mathrm{I}} \gamma_{\mathrm{P}}{ }^{\mathrm{A}}\right)$. In the mutant signaling model, PKC $\gamma$ is constitutively active and leads to phosphorylation and activation of DGK $\gamma$ in the cytosolic compartment. This occurs even during basal conditions. In this model, depolarization-induced stimulation leads to local DAG generation in the membrane compartment. This leads to cytosolto-membrane translocation of active PKC $\gamma$ and DGK $\gamma$. Once in the membrane compartment, already active and phosphorylated DGK $\gamma$ stimulates DAG metabolism. DAG levels are quickly reduced by the molecule's conversion to PA. Once DAG levels in the membrane drop, both molecules relocate back to the cytosol.

In contrast, Figure 2 shows that $\mathrm{PKC} \gamma$ can reside in one of four states in the wild-type model (Supplementary Material S2: Biochemical Reactions describing fast kinetics wildtype model). The four states are cytosolic dormant $\left(\mathrm{PKC} \gamma_{\mathrm{II}}\right)$, inactive membrane $\left(\mathrm{PKC} \gamma_{\mathrm{I}}\right)$, active membrane $\left(\mathrm{PKC} \gamma_{\mathrm{I}}{ }^{\mathrm{A}}\right)$, and active cytosolic $\left(\mathrm{PKC} \gamma_{\mathrm{II}}{ }^{\mathrm{A}}\right)$. In the mutant cascade, $\mathrm{PKC} \gamma$ exists in only two distinct forms. This is because PKC $\gamma$ is inactive in the wild-type model in its dormant cytosolic state, but constitutively active in the mutant model while residing in the cytosol. This difference leads to a reduced PKC $\gamma$ cycle in the mutant model (Figures 1, 2). Interestingly, cytosolic DGK $\gamma$ in the wild-type model is inactive, and only phosphorylated and activated in the membrane compartment. In contrast, in the mutant model, it is activated and phosphorylated in the cytosol by constitutively active PKC $\gamma$. The two-state mutant model and four-state wild-type model of PKC $\gamma$ (Figure 2) are only simplistic descriptions of a complex PKC $\gamma$ cycling process. A more realistic, complex PKC $\gamma$ cycling model should account for molecular events like translocation, binding, activation, and re-translocation of active $\mathrm{PKC} \gamma$ to the cytosol. In addition, it should include deactivation of PKC $\gamma$ back to its dormant form in the cytosol. While the processes modeled in this manuscript are complex, this study approximates the processes using simpler biochemical kinetic events that preserve key qualitative features. Cytosol-to-membrane translocation of both PKC $\gamma$ and DGK $\gamma$ in these mutant and wild-type simulations is described through proportionality functions of DAG concentrations. In addition, this study assumes non-negligible basal levels of PKC $^{\text {Active }} \gamma_{\text {II-Mutant }}(100 \mathrm{pg} / \mathrm{ml})$ and DGK $\mathrm{II} \gamma(100 \mathrm{pg} / \mathrm{ml})$ in the mutant and $\mathrm{PKC} \gamma_{\mathrm{II}}(100 \mathrm{pg} / \mathrm{ml})$ and DGK $\mathrm{II} \gamma(100 \mathrm{pg} / \mathrm{ml})$ in the wild-type model. In contrast, the basal concentration of all other forms of PKC $\gamma$ and DGK $\gamma$ is negligible. The biochemical reaction and translocation rates of this molecular loop were obtained by fitting the depolarization-induced temporal dynamics of

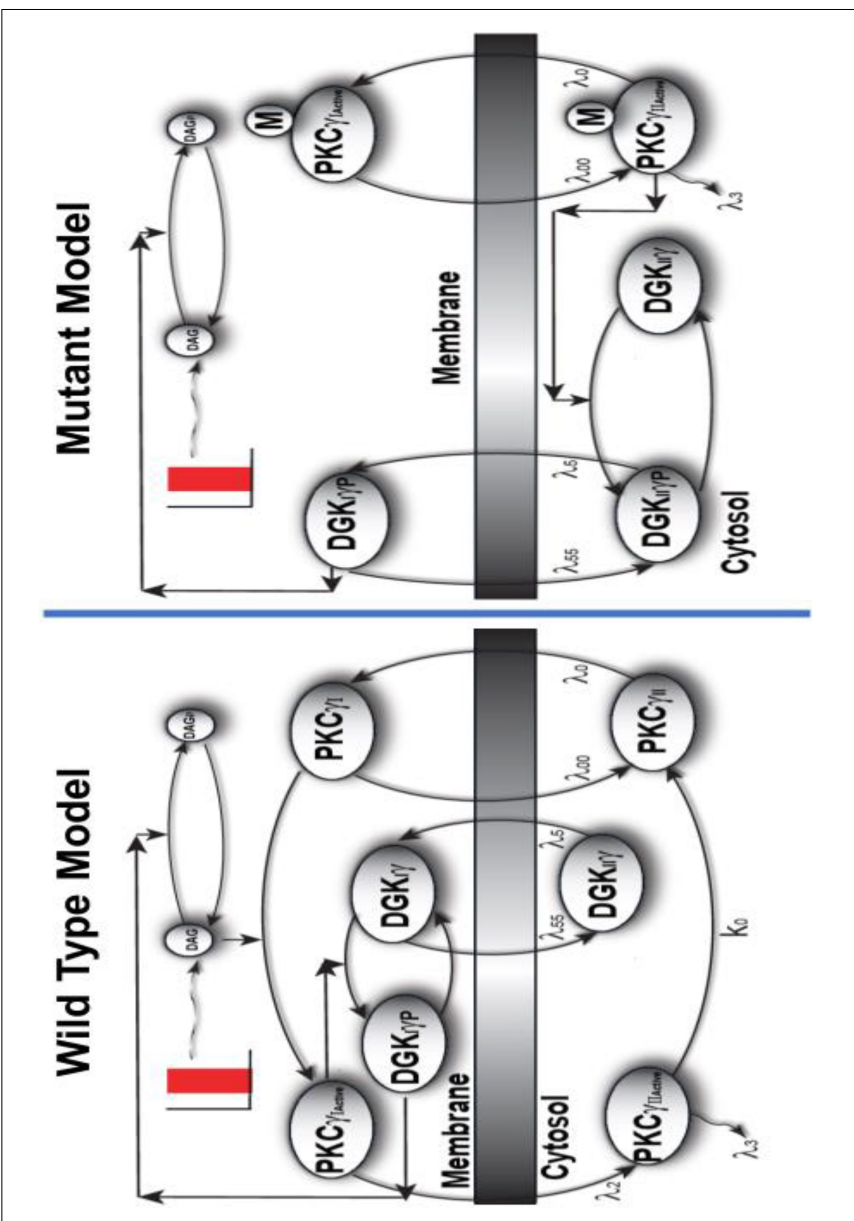

FIGURE 2 | The comparison of two-compartment, wild-type, and mutant models of local DAG signaling in PCs. Depolarization-induced local generation of DAG in PCs, in turn, leads to the functional coupling between PKC $\gamma$ and DGK $\gamma$ molecules. The wild-type model is characterized by dormant and inactive PKC $\gamma$ molecule residing in cytosol, whereas the mutant model is characterized by active PKC $\gamma$ isoform in the cytosol. In the wild-type model, the depolarization-induced activation of purinergic receptor and local generation of DAG stimulates the translocation of PKC $\gamma$ and DGK $\gamma$ molecules from cytosol to membrane compartments. Once in the membrane compartment, the DAG binds with PKC $\gamma$ and activates it, which, in turn, activates DGK $\gamma$ through phosphorylation. In the membrane compartment, the phosphorylated and active DGK $\gamma$ molecule stimulates the DAG metabolism, thus restricting its own activation. Once DAG is converted to PA in membrane compartment, both these molecules return to their dormant forms in cytosol. In contrast, the signaling in the mutant model is reduced as in the mutant model of PCs; the PKC $\gamma$ is in constitutively active state and leads to the phosphorylation and activation of DGK $\gamma$ in the cytosol even during unstimulated conditions. On stimulation, as DAG is generated in the membrane compartment, both these molecules migrate to membrane where the already activated DGK $\gamma$ molecule converts DAG to PA. It seems that signaling in the mutant model is reduced due to the constitutively active form of PKC $\gamma$ in cytosol.

PKC $\gamma$ translocation and re-translocation obtained from PCs in cerebellar slices as described in previously published studies (Shuvaev et al., 2011).

In both the mutant and wild-type models, DGK $\gamma$ inhibits its own translocation and that of PKC $\gamma$ by converting DAG into PA 
at the plasma membrane. Our models show that depolarizationinduced stimulation in the membrane compartment leads to DAG generation. DAG generation, in turn, stimulates the DGK $\gamma$ and PKC $\gamma$ translocation. In the mutant model, once the already active DGK $\gamma$ is at the plasma membrane, it directly induces DAG metabolism. In contrast, in the wildtype model, DGK $\gamma$ undergoes an activation event before becoming competent to metabolize DAG. These events restore DAG homeostasis, thus reducing migration to the membrane compartment. In both these models, local DAG generation is counterbalanced by DGK $\gamma$-assisted metabolism in the membrane compartment.

Regulation of depolarization-induced activation of G proteincoupled receptor (GPCR) pathways is a complex process (Shuvaev et al., 2011). GPCR agonist mGluR1 stimulates PLCmediated hydrolysis of phosphatidylinositol 4,5-biphosphate to produce inositol triphosphate $\left(\mathrm{IP}_{3}\right)$ and DAG (Shuvaev et al., 2011). In this study, although we are primarily focusing on the downstream signaling of the purinergic receptor pathway, we modeled local DAG biosynthesis in both the mutant and wildtype models by using a brief pulse of variable intensity. This pulse is used to mimic the effects of GPCR agonist mGluR1. This approach is simple in that it ignores the details of purinergic receptor-induced DAG biosynthesis. Since our primary focus is regulation of DAG homeostasis, and not DAG biosynthesis, this study employs this simple approach.

\section{Comparison of PKC $\gamma$ and DGK $\gamma$ Translocation Characteristics in Mutant and Wild-Type PC Models Through Numerical Simulations}

Next, the translocation characteristics of PKC $\gamma$ and DGK $\gamma$ in the mutant and wild-type models were compared through numerical simulations. Translocation characteristics were determined by measuring two key properties. First, translocation kinetics from cytosol to membrane were measured. Next, the membrane-tocytosol (M/C) ratio was measured. The kinetics of translocation are determined by measuring the time that both DAG effector molecules spend in the membrane compartment. During the course of simulations, the $\mathrm{M} / \mathrm{C}$ ratio is measured by computing the total amount of DAG effector molecules in the membrane and cytosolic compartment at every time step and then taking the ratio. In simulations mimicking the post depolarizationinduced receptor activation, the speed of translocation response is described using kinetic parameters. In contrast, during these numerical experiments, the intensity of this response is described using the $\mathrm{M} / \mathrm{C}$ ratio of $\mathrm{PKC} \gamma$ and $\mathrm{DGK} \gamma$ in the membrane and cytosolic compartments. In simulations mimicking both the mutant and wild-type models, the mGluR1-induced activation of GPCR and subsequent DAG generation were implemented using a brief 1-min pulse, as described in the previous section. The strength of pulse is described by an arbitrary parameter $S_{1}$. $S_{1}$ was set at an arbitrary level of 20. In the absence of a pulse (Figure 3, solid blue lines), there is no de novo DAG biosynthesis and the system is fixed in its basal state. In the basal state, both molecules reside in the cytosol with no possibility of translocation.
The stimulation-induced temporal dynamics of $\mathrm{PKC} \gamma$ in the mutant (Figure 3A, dashed green line) and wild-type (Figure 3A, dashed red line) models show two phases of translocation. The first is an early phase, in which PKC $\gamma$ migrates from the cytosol to the membrane. The second phase that follows is a resolution phase in which $\mathrm{PKC} \gamma$ relocates back to the cytosol. These results show that, for the same level of stimulation pulse, the translocation of PKC $\gamma$ from the cytosol to the membrane is faster in the mutant model compared to wild-type. In contrast, the translocation intensity, measured by the $\mathrm{M} / \mathrm{C}$ ratio of $\mathrm{PKC} \gamma$, is lower in the mutant model compared to wild-type (Figure 3A, dashed green and red line). These results show that cytosolicto-membrane molecular migration in both models is dependent on local DAG concentration at the plasma membrane and is linked to de novo DAG biosynthesis. In both the mutant and wild-type models, DAG concentration can be controlled through the duration and amplitude of pulse stimulation at the plasma membrane compartment.

In both models, the PKC $\gamma$ translocation rate is a directly proportional function of DAG concentration (Supplementary Material S1: Table S1 and Supplementary Material S3: Table S2). When the mutant model was perturbed with different levels of stimulation, the results indicate that when stimulation strength is set at moderate levels (Figure 4A, inset; pulse strength parameter $S_{1}=20$ ), only a small pool of DAG is generated at the plasma membrane. This induces a low-intensity migration event reflected by a maximal M/C ratio. The M/C ratio is linked to pulse strength (Figure 4A). Higher levels of stimulation generate (Figure 4A, inset; pulse strength parameter $S_{1}=40$ and 60) much larger pools of DAG at the plasma membrane. This induces migration of a large pool of PKC $\gamma$ to the membrane. Interestingly, perturbation of the wild-type model (Figure 4B) also generates the same pattern with regard to the $\mathrm{M} / \mathrm{C}$ ratio of PKC $\gamma$. However, at higher stimulation strengths, the maximal $\mathrm{M} / \mathrm{C}$ ratio of $\mathrm{PKC} \gamma$ in the wild-type model is five-times more than the ratio at moderate stimulation. In contrast, it is 10-times more at even higher stimulation strength, showing the effects of DAG generation on translocation of $\mathrm{PKC} \gamma$ from the cytosol to the membrane.

In this study, DGK $\gamma$ translocation is spatially similar, but temporally distinct, from $\mathrm{PKC} \gamma$. The migration of DGK $\gamma$ is also controlled by DAG concentrations at the plasma membrane. The sensitivity of this migratory event is different from that of PKC $\gamma$. DGK $\gamma$ translocation is also described by a proportionality function (Supplementary Material S1: Table S1 and Supplementary Material S3: Table S2) of DAG concentration at the plasma membrane. Compared to PKC $\gamma$, however, the slope of this function is much smaller. This means that DGK $\gamma$ migration from the cytosol to the plasma membrane requires a much larger increase in DAG concentration at the plasma membrane. This approach was adopted so that the proposed models align with previous experimental observations showing different translocation sensitivities of $\mathrm{PKC} \gamma$ and $\mathrm{DGK} \gamma$ to $\mathrm{Ca}^{+2}$ concentrations (Yamaguchi et al., 2006). These observations suggest that both PKC $\gamma$ and DGK $\gamma$ possess DAG-sensitive $\mathrm{C}_{1}$ and $\mathrm{Ca}^{+2}$ sensitive $\mathrm{C}_{2}$ domain. Interestingly, nanomolar elevation in 

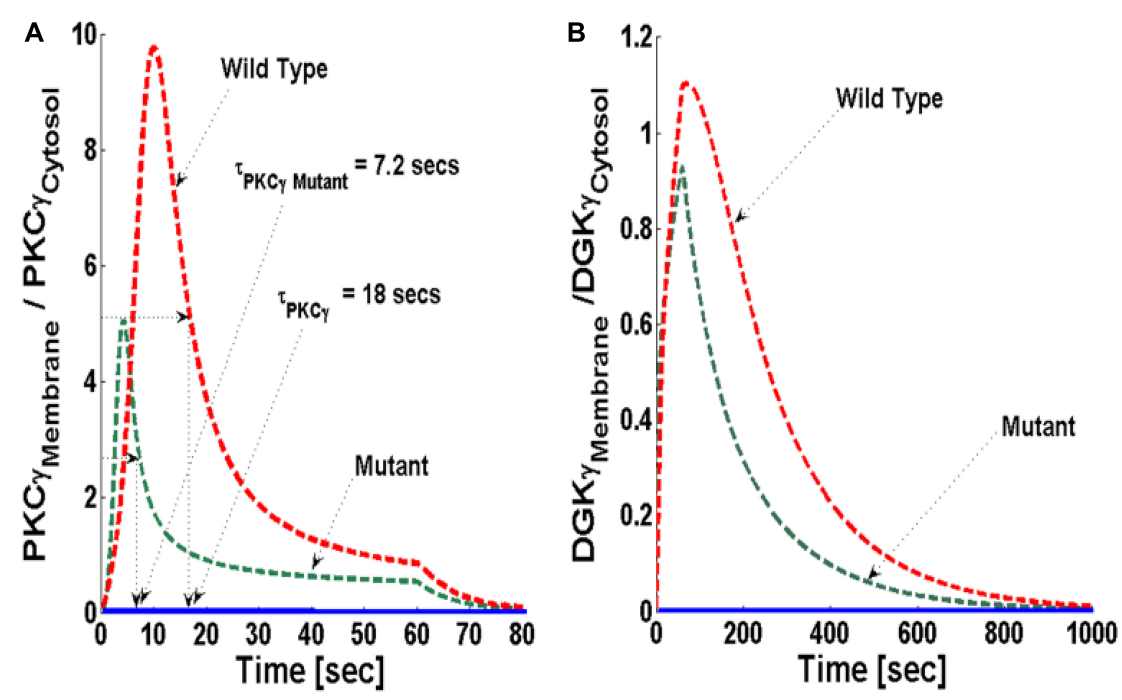

FIGURE 3 | The simulations mimicking the comparison of depolarization-induced translocation of PKC $\gamma$ and DGK $\gamma$ molecules in the mutant and wild-type models of PCs. These results show the membrane-to-cytosol (M/C) ratio of PKC $\gamma$ and DGK $\gamma$ molecules in response to a brief 1-min pulse, which leads to the rapid generation of DAG in the membrane compartment. Here, the strength of stimulation is controlled by setting the pulse parameter " $S_{1}$ " at 20 . The generation of the second messenger, in turn, stimulates the translocation of both PKC $\gamma$ and DGK $\gamma$ from cytosol to membrane. Here, the solid line represents the non-stimulation and the dashed line represents the stimulation condition (green dashed line, mutant; red dashed line, wild-type PCs). (A) The translocation characteristics of the PKC $\gamma$ molecule in both mutant and wild-type models. These results suggest that for identical strength and duration of stimulation, the cytosol-to-membrane migration kinetics of PKC $\gamma$ molecule are much faster in mutant models compared to wild-types. Compared to wild-types, the membrane residence time of PKC $\gamma$ molecule is shorter in mutant models, i.e., $7.2 \mathrm{~s}$ for mutant and $18 \mathrm{~s}$ for wild-type models. (B) The translocation characteristics of the DGK $\gamma$ molecule in both mutant and wild-type models. These results show that the membrane residence time of the DGK $\gamma$ molecule is shorter in mutant models compared to models representing wild-type PCs.

$\mathrm{Ca}^{+2}$ concentration was enough to warrant $\mathrm{PKC} \gamma$ translocation, whereas DGK $\gamma$ translocation required micromolar increase in $\mathrm{Ca}^{+2}$ concentration (Yamaguchi et al., 2006).

At the plasma membrane, the coupling between PKC $\gamma$ and DGK $\gamma$ can be quantified by measuring colocalization time between these two molecules. Colocalization time is defined as the duration for which these molecules may interact with each other. This time interval also determines the duration during which a negative feedback loop is functional and effectively facilitating DAG metabolism. Colocalization time depends on DAG concentration in the plasma membrane, re-translocation rates of the inactive molecules, the remigration rate of active PKC $\gamma$, the activation rate and DAG binding activity of PKC $\gamma$, and activation and phosphorylation of DGK $\gamma$.

In order to estimate the parameters of proposed PKC $\gamma$ and DGK $\gamma$ interaction in PCs, we fixed the membrane residence duration of PKC $\gamma$ in both mutant and wild-type models well within the experimentally reported ranges (Shuvaev et al., 2011) and determined the unknown parameters of molecular loop as well as the translocation and remigration rates of DGK $\gamma$. We also assumed that, similar to the $\mathrm{CHO}$ cell model, the sensitivity of PKC $\gamma$ translocation to DAG concentration is higher than DGK $\gamma$ (Supplementary Material S1: Table S1 and Supplementary Material S3: Table S2). We calibrated our models by matching the ranges of membrane residence duration of PKC $\gamma$ in mutant and wild-type models of cPCs (Shuvaev et al., 2011). This ad hoc approach could be questioned, but in the absence of direct translocation data of DGK $\gamma$ migration and remigration in PCs, we believe that this is a reasonable approximation. The assumption made here should be tested directly and the translocation rate of DGK $\gamma$ should be measured directly in PCs. However, it is unlikely that the overall structure of this model and the conclusion drawn from this model will be significantly different based on these assumptions.

\section{Simulations Mimicking the Effect of the Rate of DAG Phosphorylation on the Translocation Characteristics of PKC $\gamma$ in Mutant and Wild-Type Models of PCs}

The residence time of $\mathrm{PKC} \gamma$ at the membrane is modulated by DAG levels. DAG metabolism at the membrane is regulated by active and phosphorylated DGK $\gamma$. DGK $\gamma$ leads to DAG phosphorylation, an essential step for the conversion of DAG to PA. It is possible that the molecular events involved in DAG phosphorylation may influence the residence time of PKC $\gamma$ at the membrane. This study addresses the question of how the DAG phosphorylation rate, " $\mathrm{k}_{6}$ " in the mutant model and " $\mathrm{k}_{8}$ " in the wild-type model, might influence the intensity and translocation of PKC $\gamma$ and DGK $\gamma$ from the cytosol to the membrane. The parameter " $k_{6}$ " in the mutant and " $k_{8}$ " in the wild-type model describe the feed forward rate constant of DAG phosphorylation by DGK $\gamma_{P}$. This study tested the hypothesis that how an increase in parameters $\mathrm{k}_{6}$ and $\mathrm{k}_{8}$ could influence the translocation as well as membrane residence duration of PKC $\gamma$. The simulations compared three difference cases: (a) baseline $\mathrm{k}_{6}$ and $\mathrm{k}_{8}\left(\mathrm{k}_{6}=0.95\right.$ 

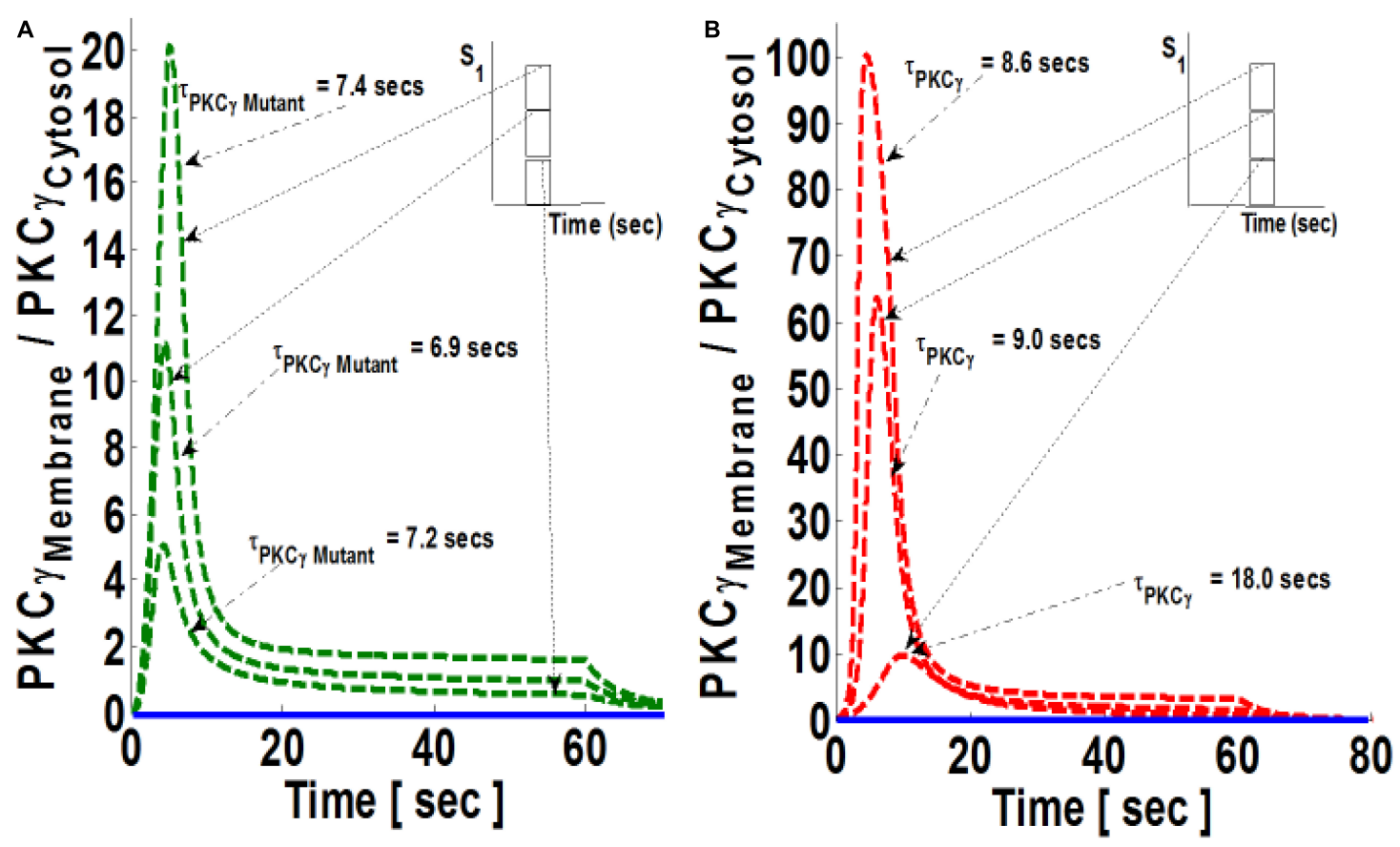

FIGURE 4 | The simulations mimicking the effect of stimulation strength on the comparative translocation kinetics of PKC $\gamma$ isoform in the mutant and wild-type models of PCs. Here, the strength of stimulation is controlled by setting the pulse parameter " $\mathrm{S}_{1}$ " at different levels. The parameter $\mathrm{S}_{1}$ is set at arbitrary values of 20 , 40, and 60 (inset). The duration of pulse for all these three cases is $1 \mathrm{~min}$. Here, the solid line represents the non-stimulation and the dashed line represents the stimulation condition (green dashed line, mutant; red dashed line, wild-type PCs). (A) Translocation characteristics of PKC $\gamma$ in the mutant model. Here, results show that maximum levels of $\mathrm{M} / \mathrm{C}$ ratio of $\mathrm{PKC} \gamma$ increases with increase in the strength of parameter " $\mathrm{S}_{1}$ "; however, the residence time of mutant PKC $\gamma$ first decreases and then increases with pulse strength $\left(S_{1}=20\right.$, leads to maximum M/C levels of 5 and $\tau_{P K C_{\gamma} \text { mutant }}=7.2 \mathrm{~s} ; \mathrm{S}_{1}=40$, leads to maximum $\mathrm{M} / \mathrm{C}$ levels of 11 and $\tau_{\mathrm{PKC}_{\gamma} \text { mutant }}=6.9 \mathrm{~s} ; \mathrm{S}_{1}=60$, leads to maximum M/C levels of 20 and $\tau_{\mathrm{PKC}_{\gamma} \text { mutant }}=7.4 \mathrm{~s}$ ). (B) Translocation characteristics of PKC $\gamma$ in the wild-type models. Here, results show that maximum levels of the $\mathrm{M} / \mathrm{C}$ ratio of $\mathrm{PKC} \gamma$ increases with increase in the strength of parameter "S ${ }_{1}$," and the residence time of wild-type PKC $\gamma$ decreases with pulse strength $\left(\mathrm{S}_{1}=20\right.$, leads to maximum $\mathrm{M} / \mathrm{C}$ levels of 10 and $\tau_{\mathrm{PKC}}=18 \mathrm{~s} ; \mathrm{S}_{1}=40$, leads to maximum $\mathrm{M} / \mathrm{C}$ levels of 64 and $\tau_{\mathrm{PKC}}=9 \mathrm{~s}$; $\mathrm{S}_{1}=60$, leads to maximum $\mathrm{M} / \mathrm{C}$ levels of 100 and $\left.\tau_{\mathrm{PKC}} \mathrm{C}_{\gamma}=8.6 \mathrm{~s}\right)$.

$\mathrm{pM}^{-1} \mathrm{~s}^{-1}$ and $\mathrm{k}_{8}=0.95 \mathrm{pM}^{-1} \mathrm{~s}^{-1}$ ); (b) 25-times increase in the $\mathrm{k}_{6}$ and $\mathrm{k}_{8}$; (c) 100-times increase in the $\mathrm{k}_{6}$ and $\mathrm{k}_{8}$. The results show that increasing the parameter $\mathrm{k}_{6}$ not only reduces the intensity of translocation but also the membrane residence time of PKC $\gamma$ in the mutant model (Figure 5A). A 25-fold increase in $\mathrm{k}_{6}$ causes the maximum $\mathrm{M} / \mathrm{C}$ ratio to decrease $10 \%$. In contrast, membrane residence time decreased $13 \%$ in the mutant model (Figure 5A). Interestingly, increasing $\mathrm{k}_{6}$ from 25 - to 100 -fold had no effect on translocation or membrane residence duration for PKC $\gamma$ in the mutant model (Figure 5A). The results from the wild-type model show that a 25 -fold increase in $\mathrm{k}_{8}$ reduces $\mathrm{PKC} \gamma$ translocation intensity to almost $40 \%$. In contrast, membrane residence time is reduced by only 11\% (Figure 5B). Furthermore, an increase in $\mathrm{k}_{8}$ from 25 - to 100 -fold had an almost negligible influence on membrane residence duration and the magnitude of translocation (Figure 5B).

Next, this study investigated how blocking DAG phosphorylation influences the magnitude of $\mathrm{PKC} \gamma$ translocation as well as PKC $\gamma$ membrane residence duration in both the mutant and wild-type models. Our results indicate that blocking parameter $\mathrm{k}_{6}$ in the mutant model increases both the magnitude and membrane residence duration of $\mathrm{PKC \gamma}$ (Figure 6). Our results show that 90 and $95 \%$ blocking of $\mathrm{k}_{6}$ in the mutant model leads to maximum $\mathrm{M} / \mathrm{C}$ ratios of 8.8 and 12.2 , respectively. In contrast, $\mathrm{PKC} \gamma$ membrane residence duration times are 10.5 and $14.5 \mathrm{~s}$, respectively (Figure 6A). These results indicate that 95\% blocking in the mutant model leads to an almost twofold increase in the magnitude of translocation and membrane residence duration of $\mathrm{PKC} \gamma$ (Figure 6A). Similarly, our results indicate that blocking the DAG phosphorylation parameter, $\mathrm{k}_{8}$, in wild-type models also increases translocation intensity and membrane residence duration (Figure 6B). Our results show that 90 and $95 \%$ blocking of $\mathrm{k}_{8}$ in the wild-type model leads to 3 and 5 -fold increases in the magnitude of translocation (Figure 6B; measured as maximum in $\mathrm{M} / \mathrm{C}$ ratio). In contrast, only 5 and $10 \%$ increases in membrane residence duration were observed for the wild-type model of PKC $\gamma$ in $\mathrm{CPCs}$ (Figure 6B).

\section{Simulations Mimicking the Influence of the DGK $\gamma$ Activation Rate on the Translocation Characteristics of PKC $\gamma$}

DGK $\gamma$ phosphorylation and activation play key roles in regulating DAG homeostasis in the mutant and wild-type models. In the mutant model, DGK $\gamma$ activation takes place in the cytosol. In contrast, activation takes place in the 

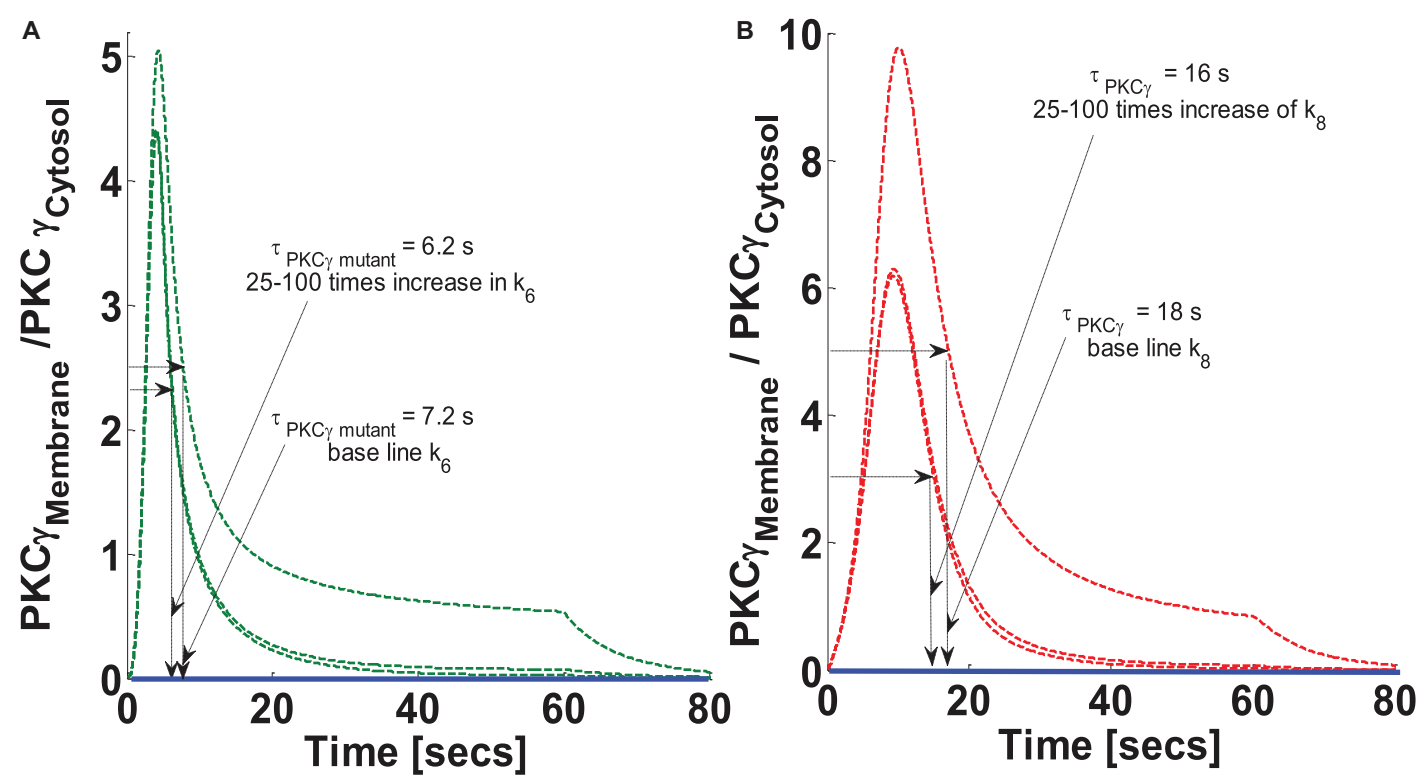

FIGURE 5 | The simulations mimicking the effect of feed forward rate constant of DAG phosphorylation by DGK $\gamma_{P}$ on the comparative translocation kinetics of PKC $\gamma$ molecule in the mutant and wild-type models of PCs. Parameter $\mathrm{k}_{6}$ represent this rate constant in mutant models, whereas in wild-types, it is represented by $\mathrm{k}_{8}$. Here, the strength of stimulation is controlled by setting the pulse parameter " $\mathrm{S}_{1}$ " at 20 . The duration of pulse is set for 1 min. Here, the solid line represents the non-stimulation and the dashed line represents the stimulation condition (green dashed line, mutant; red dashed line, wild-type PCs). (A) Translocation characteristics of PKC $\gamma$ in the mutant model. Here, results show that increasing the parameter $\mathrm{k}_{6}$ in the mutant model reduces the membrane residence time of $\mathrm{PKC}_{\gamma}$ (at baseline, $\mathrm{k}_{6}, \tau_{\mathrm{PKC}_{\gamma} \text { mutant }}=7.2 \mathrm{~s}$; and $25-100$ times increase in $\mathrm{k}_{6}$ leads to $\tau_{\mathrm{PKC}} \mathrm{C}_{\text {mutant }}=6.2 \mathrm{~s}$ ). (B) Translocation characteristics of PKC $\gamma$ in the wild-type models. Here, results show that increasing the parameter $\mathrm{k}_{8}$ in the wild-type model reduces the membrane residence time of PKC $\gamma$ (at baseline, $\mathrm{k}_{8}$, $\tau_{\mathrm{PKC}}=18 \mathrm{~s}$; and $25-100$ times increase in $\mathrm{k}_{8}$ leads to $\left.\tau_{\mathrm{PKC}_{\gamma}}=16 \mathrm{~s}\right)$.

membrane compartment in the wild-type model. Next, through numerical simulations, this study investigated how reducing DGK $\gamma$ activation and phosphorylation rates may influence the magnitude of translocation and the membrane residence duration of $\mathrm{PKC} \gamma$ in the mutant and wild-type models. Our results show that blocking parameter $\mathrm{k}_{4}$ in the mutant model reduces the magnitude of $\mathrm{PKC} \gamma$ translocation, but increases membrane residence duration (Figure 7A). The results show that $80 \%$ blocking results in a $70 \%$ reduction in translocation magnitude and a near 2.5-fold increase in membrane residence time (Figure 7B). Furthermore, 90\% blocking results in an $80 \%$ reduction in the magnitude of translocation and an almost threefold increase in membrane residence time of PKC $\gamma$ (Figure 7A). Interestingly, the results from the wild-type model show that $80 \%$ blocking increases magnitude by fivefold and reduces membrane residence time by 33\% (Figure 7B). Furthermore, $90 \%$ blocking increases magnitude by 12 -fold and reduces duration by $22 \%$ (Figure $7 \mathbf{B}$ ) compared to the baseline case of no blocking.

\section{How Can the PKC $\gamma$-to-DGK $\gamma$ Expression Ratio Influence the Translocation Characteristics of PKC $\gamma$ ?}

The formulation of both models indicates that DAG levels, after stimulation, are controlled through a net negative feedback loop between its effector molecules PKC $\gamma$ and DGK $\gamma$. One intriguing and still-unanswered question is how the relative expression of these molecules may influence negative feedback efficacy. In this simulation, it was assumed that both DAG effector molecules are equally expressed in the cytosol (choice of equal expressions is empirical and is chosen here to have a balanced effect on negative feedback loop). This study tested how changes in the expression ratio of PKC $\gamma$ and DGK $\gamma$ may influence the magnitude of $\mathrm{PKC} \gamma$ translocation, as well as its residence time, in the membrane compartment. We selected three different ratios to test. The ratios were PKC $\gamma:$ DGK $\gamma$ 1:1, PKC $\gamma: D G K \gamma$ 1:0.5, and PKC $\gamma: D G K \gamma$ 1:0.3. In both models, the systems are perturbed by a brief pulse $\left(S_{1}=7\right)$ which leads to rapid DAG generation in the membrane compartment. Our results show that reducing DGK $\gamma$ expression in the mutant model increases the magnitude of PKC $\gamma$ translocation as well as the membrane residence time (Figure 8A). It is possible that reducing DGK $\gamma$ expression in the mutant model reduces the efficacy of negative feedback. Furthermore, our results indicate that reducing DGK $\gamma$ expression in the wild-type models also reduces the efficacy of negative feedback. This results in increased translocation magnitude as well as increased membrane residence time of PKC $\gamma$ (Figure 8B).

\section{DISCUSSION}

Recent evidence links mutations in the $\mathrm{C}_{1}$ domain of PKC $\gamma$ (Yabe et al., 2003; Shimobayashi, 2016) to cerebellar neurodegeneration in SCA14 disease. It is likely that a gain of function is the mechanism causing the observed neuronal degeneration that 

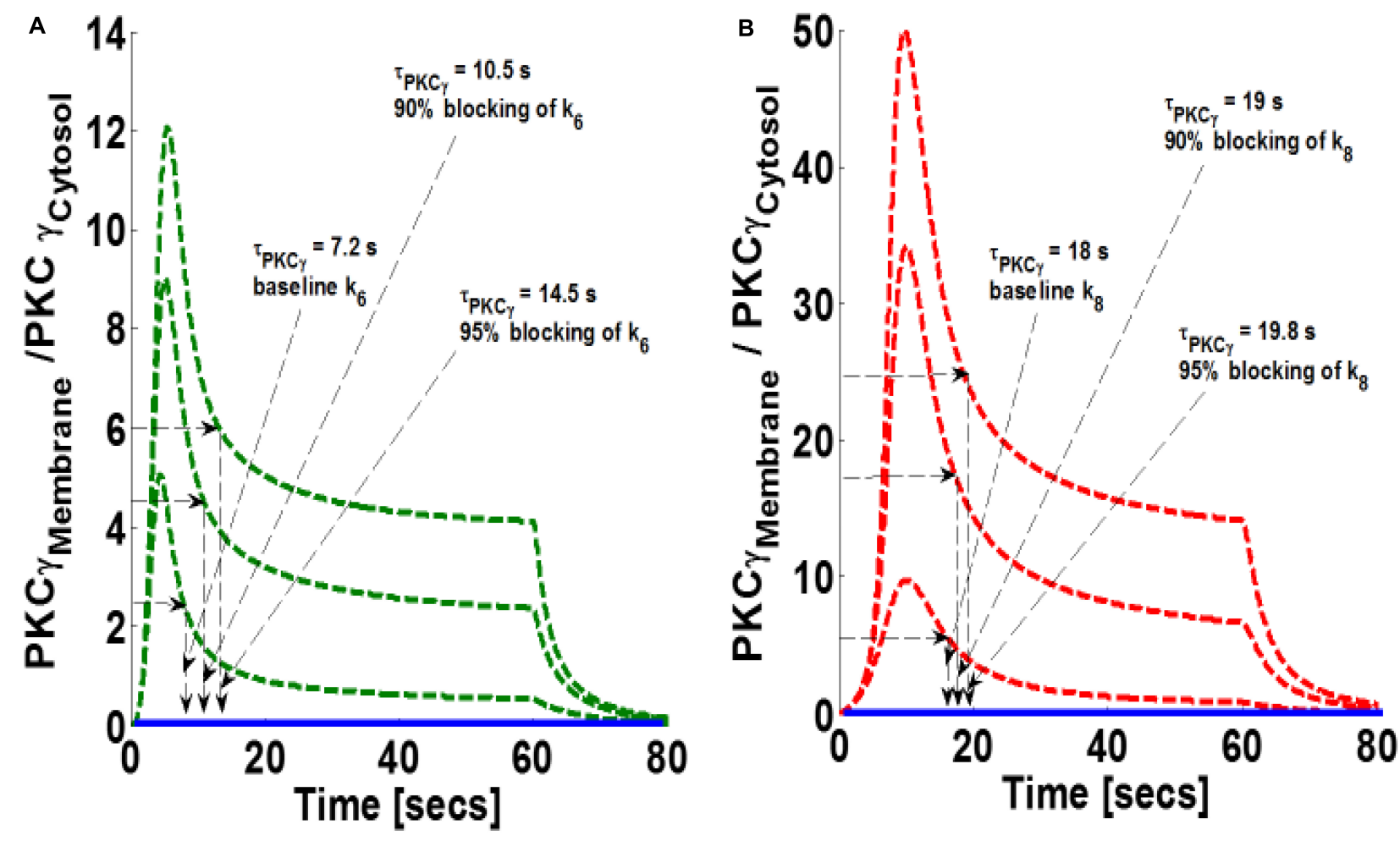

FIGURE 6 | The simulations mimicking the effect of blocking of forward rate constant of DAG phosphorylation by DGK $\gamma_{P}$ on the comparative translocation kinetics of PKC $\gamma$ molecule in the mutant and wild-type models of PCs. Parameter $\mathrm{k}_{6}$ represent this rate constant in mutant models, whereas in wild-types, it is represented by $k_{8}$. Here, the strength of stimulation is controlled by setting the pulse parameter " $S_{1}$ " at 20 . The duration of pulse is set for 1 min. Here, the solid line represents the non-stimulation and the dashed line represents the stimulation condition (green dashed line, mutant; red dashed line, wild-type PCs). (A) Translocation characteristics of PKC $\gamma$ in the mutant model. Here, results show that blocking the parameter $\mathrm{k}_{6}$ in the mutant model increases the membrane residence time of PKC $\gamma$ (baseline, $\mathrm{k}_{6}, \tau_{\mathrm{PKC}_{\gamma} \text { mutant }}=7.2 \mathrm{~s} ; 90 \%$ blocking of $\mathrm{k}_{6}$ leads to $\tau_{\mathrm{PKC}_{\gamma} \text { mutant }}=10.5 \mathrm{~s} ; 95 \%$ blocking of $\mathrm{k}_{6}$ leads to $\tau_{\mathrm{PKC}} \mathrm{m}_{\gamma}$ mutant $=14.5 \mathrm{~s}$ ). (B) Translocation characteristics of PKC $\gamma$ in the wild-type models. Here, results show that blocking the parameter $\mathrm{k}_{8}$ in the wild-type model enhances the membrane residence time of PKC $\gamma$ (baseline, $\mathrm{k}_{8}, \tau_{\mathrm{PKC}_{\gamma}}=18 \mathrm{~s} ; 90 \%$ blocking leads to $\tau_{\mathrm{PKC}}=19 \mathrm{~s} ; 95 \%$ blocking leads to $\tau_{\mathrm{PKC}}=19.8 \mathrm{~s}$ ).

occurs during SCA14. It is likely because the PKC $\gamma$ knockout failed to exhibit cerebellar degeneration and only showed slight ataxia (Chen et al., 1995; Kano et al., 1995). This was probably due to the loss of the pruning process at the PFPC synapses. Evidence also suggests that mutations in the $C_{1}$ domain could alter the activation and translocation profiles of PKC $\gamma$ (Adachi et al., 2008; Shuvaev et al., 2011; Wong et al., 2018). It could be that these alterations in the enzymological properties of $\mathrm{PKC} \gamma$ and its regulator DGK $\gamma$ are responsible for the dysregulation of $\mathrm{Ca}^{+2}$ homeostasis during SCA14 disease pathology. This disruption of $\mathrm{Ca}^{+2}$ equilibrium is probably due to altered gating characteristics of $\mathrm{TRPC}_{3}$ channels. One possible cause of $\mathrm{TRPC}_{3}$ gating dysregulation may be the inability of mutant molecules to completely phosphorylate $\mathrm{TRPC}_{3}$ channels. In this case, the $\mathrm{Ca}^{+2}$ influx during disease-related PCs was only partially blocked. Consequently, the intracellular accumulation of $\mathrm{Ca}^{+2}$ in PCs is probably an underlying cause of observed neurodegeneration in SCA14 patients (Shuvaev et al., 2011). Thus, in order to understand the mechanisms of SCA14 disease, it is essential to study the associated changes in the enzymological properties of its key molecular players (Yamaguchi et al., 2006).
This study focuses on analyzing the enzymological properties of PKC $\gamma$ and DGK $\gamma$ in the context of SCA14 disease. Here, through a computational approach, we compare the signaling interactions of PKC $\gamma$ and DGK $\gamma$ in wild-type and mutant cells. Experimental evidence shows that despite the increase in activity, the membrane residence time of PKC $\gamma$ is reduced in mutant models of PCs compared to wild-type models (Adachi et al., 2008; Shuvaev et al., 2011). Interestingly, another set of observations indicate that despite constitutive activity in cytosol, the activity of mutant PKC $\gamma$ is reduced in the membrane compartment (Verbeek et al., 2008). In turn, this reduction in membrane residence duration or activity of $\mathrm{PKC} \gamma$ is believed to be linked to insufficient phosphorylation of $\mathrm{TRPC}_{3}$. This causes a disruption in $\mathrm{Ca}^{+2}$ homeostasis (Adachi et al., 2008; Henning, 2011; Shuvaev et al., 2011). Here, we investigate the key question of why membrane residence time of $\mathrm{PKC} \gamma$ decreases in SCA14 disease. This study investigates this question by proposing and numerically evaluating two contrasting signaling models of depolarization-induced DAG generation and regulation in both wild-type and mutant PCs. The models compare the possible signaling cascades of DAG both in the SCA14 mutant and in wild-type PCs through simulations. 

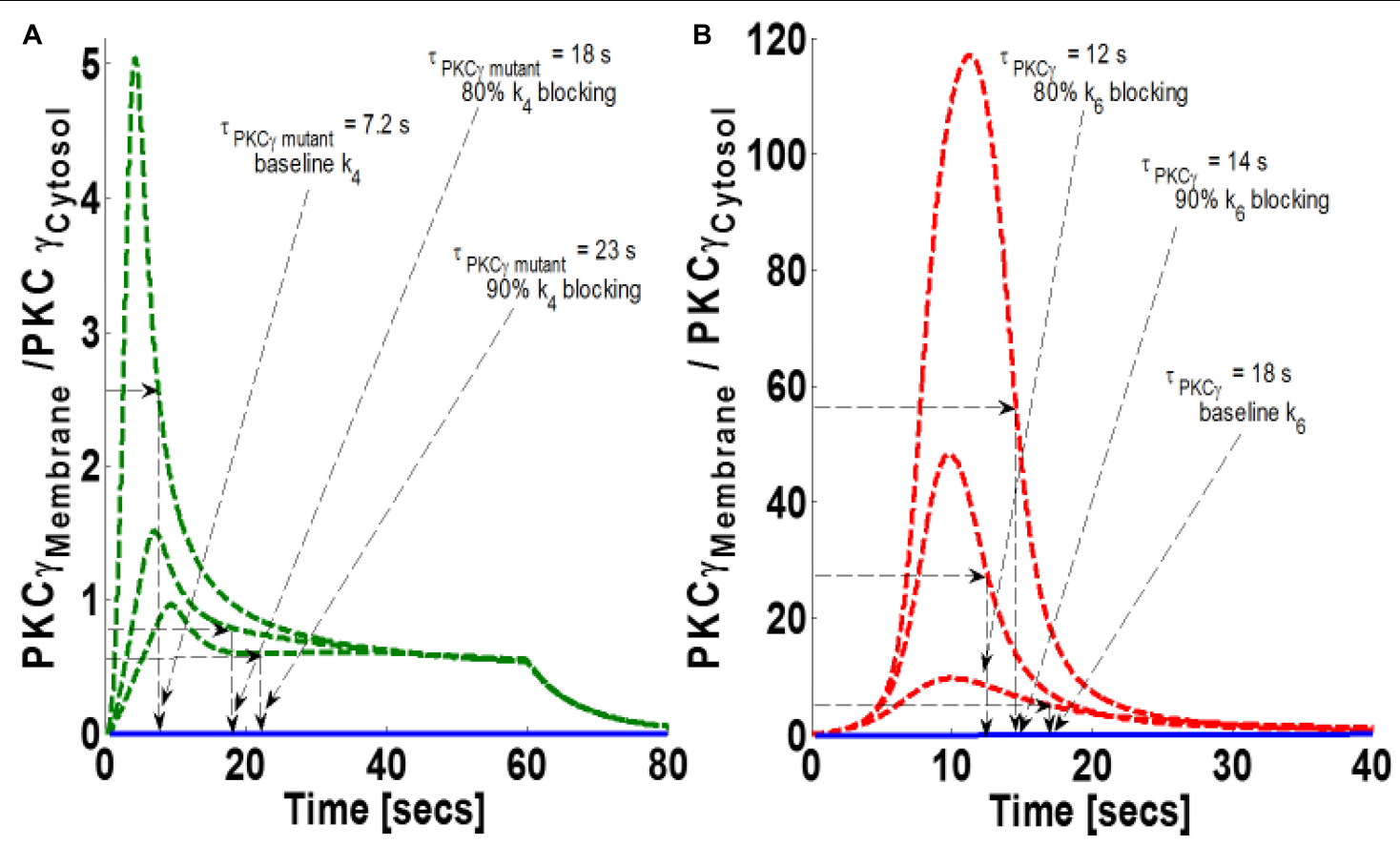

FIGURE 7 | The simulations mimicking the effect of blocking the rate constant of DGK $\gamma$ phosphorylation to DGK $\gamma_{P}$ on the comparative translocation kinetics of $\mathrm{PKC} \gamma$ molecule in the mutant and wild-type models of PCs. Parameter $\mathrm{k}_{4}$ represents this rate constant in mutant models, whereas in wild-types, it is represented by $k_{6}$. Here, the strength of stimulation is controlled by setting the pulse parameter " $S_{1}$ " at 20 . The duration of pulse is set for 1 min; the solid line represents the non-stimulation and the dashed line represents the stimulation condition (green dashed line, mutant; red dashed line, wild-type PCs). (A) Translocation characteristics of $\mathrm{PKC} \gamma$ in the mutant model. Here, results show that blocking the parameter $\mathrm{k}_{4}$ in the mutant model increases the membrane residence time of PKC $\gamma$ (baseline, $\mathrm{k}_{4}, \tau_{\mathrm{PKC}} \mathrm{Pmutant}=7.2 \mathrm{~s} ; 80 \%$ blocking of $\mathrm{k}_{4}$ leads to $\tau_{\mathrm{PKC}} \mathrm{C}_{\gamma}$ mutant $=18 \mathrm{~s} ; 90 \%$ blocking of $\mathrm{k}_{4}$ leads to $\tau_{\mathrm{PKC}} \mathrm{l}_{\mathrm{mutant}}=23 \mathrm{~s}$ ). (B) Translocation characteristics of $\mathrm{PKC} \gamma$ in the wild-type models. Here, results show that blocking the parameter $\mathrm{k}_{8}$ in the wild-type model reduces the membrane residence time of PKC $\gamma$ (baseline, $\mathrm{k}_{6}, \tau_{\mathrm{PKC}_{\gamma}}=18 \mathrm{~s} ; 80 \%$ blocking leads to $\tau_{\mathrm{PKC}_{\gamma}}=12 \mathrm{~s} ; 90 \%$ blocking leads to $\tau_{\mathrm{PKC}}=14 \mathrm{~s}$ ).

Precise modulation of $\mathrm{TRPC}_{3}$ gating is essential for the viability of PCs and their characteristically dense dendritic tree (Shuvaev et al., 2011; Henning, 2011). Evidence also suggests that, in a single PC, the $\mathrm{TRPC}_{3}$ channels are by far the most abundant molecule compared to the other members of the TRPC family (Henning, 2011). TRPC 3 proteins are mainly found in the PC soma and dendrites (Henning, 2011). These channels are regulated downstream of $\mathrm{mGluR}_{1}$ pathways and play a key role in the maintenance of calcium homeostasis in cPCs (Henning, 2011). Furthermore, experimental evidence suggests that precise gating of the $\mathrm{TRPC}_{3}$ channel is modulated by $\mathrm{PKC} \gamma$ in the membrane compartment (Henning, 2011; Shuvaev et al., 2011; Shimobayashi, 2016). Evidence also suggests that PKC $\gamma$ may modulate phosphorylation and hence inactivation of the $\mathrm{TRPC}_{3}$ channel. Thus, membrane residence duration of $\mathrm{PKC} \gamma$ may play a critical role in regulating channel gating mechanisms. The membrane residence time of $\mathrm{PKC} \gamma$ is regulated by $\mathrm{DAG}$, and, as DAG is converted to PA by DGK $\gamma$, membrane-bound PKC returns to the cytosol (Shuvaev et al., 2011).

The simulations in this study focused on the key question of how reduced membrane residence time of mutant PKC $\gamma$ is based on comparing mutant and wild-type models under the same conditions. The wild-type model within this study is based on stimulation-induced translocation and activation of PKC $\gamma$ and DGK $\gamma$. The mutant model, however, is based on constitutively active PKC $\gamma$ in the cytosol (Wong et al., 2018) and activation and phosphorylation of DGK $\gamma$ even during basal conditions. In the mutant model, stimulation-induced translocation of both active molecules from the cytosol to the plasma membrane leads to quick and direct metabolism of DAG in the plasma membrane compartment. Here, all the parameters in both models are set at the same numerical values and both the models are perturbed with a similar strength pulse. The goal of this is to test what happens to the membrane residence duration within two different signaling topologies. If all the kinetics, translocation, and stimulation strength parameters are kept the same, how does $\tau_{\mathrm{PKC}_{\gamma}}$ change in the wild-type and mutant models of cPCs? Here, $\tau_{\mathrm{PKC}_{\gamma}}$ is the membrane residence time of PKC $\gamma$ and is defined as average residence duration during which 50\% of membrane $\mathrm{PKC} \gamma$ has re-translocated back to cytosol. Interestingly, the results show that $\tau_{\mathrm{PKC}_{\gamma}}=18 \mathrm{~s}$ for the wild-type model and $\tau_{\mathrm{PKC}_{\gamma}}=7.2 \mathrm{~s}$ for the mutant model. Indeed, these results elucidate that alteration of topological structure between two models could lead to differences in membrane residence time. The results here indicate that $\mathrm{PKC} \gamma$ residence time in the mutant model is approximately threefold shorter than in the wild-type. These results are consistent with previous experimental recordings in PCs from cerebellar slices $\left(\tau_{\mathrm{PKC}}=19 \mathrm{~s}\right.$ for wild-type cells and $6.0 \mathrm{~s}$ for mutant cells) (Shuvaev et al., 2011). These results support the hypothesis 

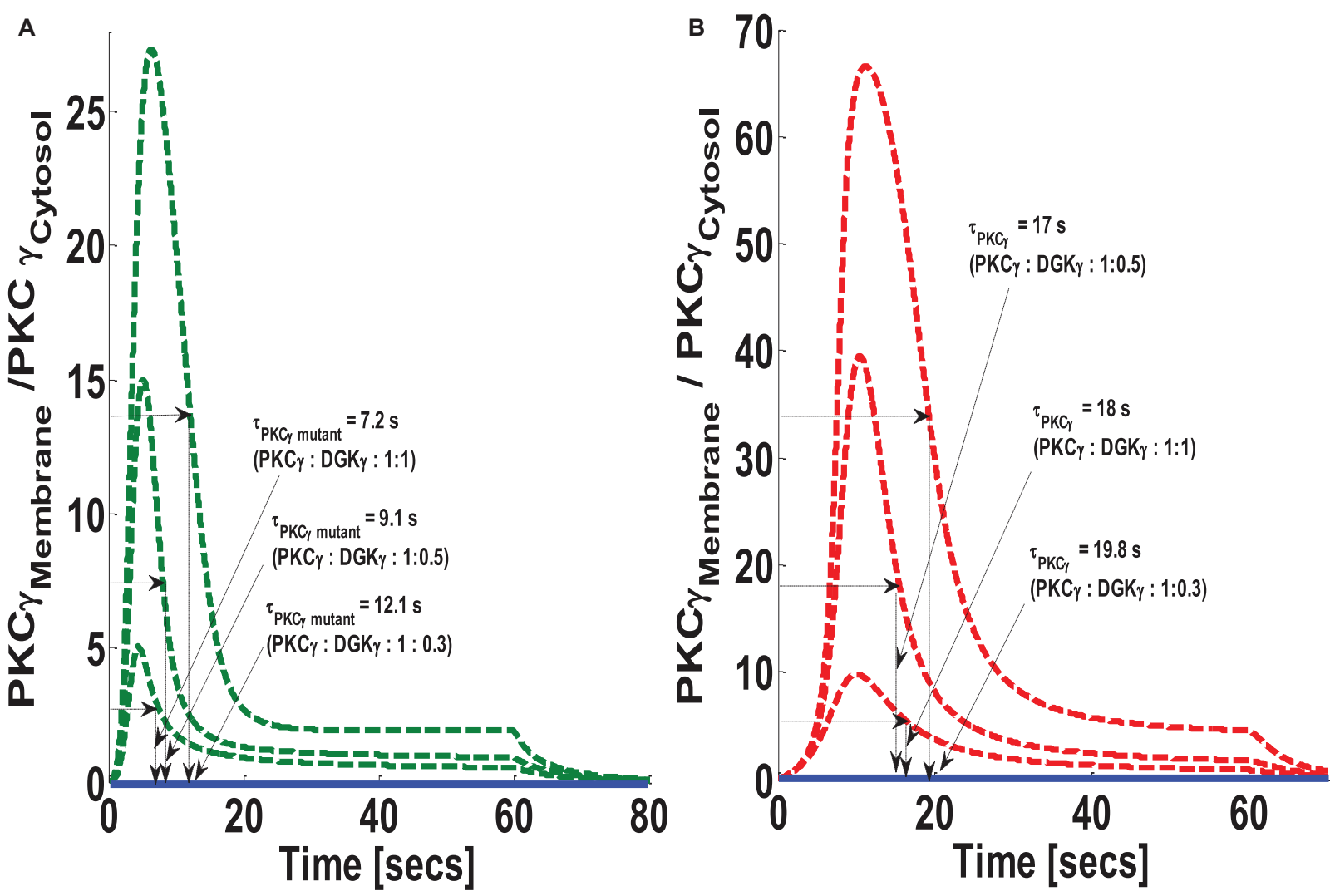

FIGURE 8| The simulations mimicking the effect of PKC $\gamma$ to DGK $\gamma$ expression ratio on the comparative translocation kinetics of PKC $\gamma$ molecule in the mutant and wild-type models of PCs. Three different levels of expression ratios are used (PKC $\gamma: D G K \gamma: 1: 1 ;$ PKC $\gamma: D G K \gamma: 1: 0.5 ;$ PKC $\gamma: D G K \gamma: 1: 0.3)$. Here, the strength of stimulation is controlled by setting the pulse parameter " $S_{1}$ " at 20 . The duration of pulse is set for 1 min; the solid line represents the non-stimulation and the dashed line represents the stimulation condition (green dashed line, mutant; red dashed line, wild-type PCs). (A) Translocation characteristics of PKC $\gamma$ in the mutant model. Here, results indicate that reducing the PKC $\gamma$ :DGK $\gamma$ expression ratio increases the membrane residence time of mutant $\gamma$ isoform (PKC $\gamma$ :DGK $\gamma$ : 1:1,

$\tau_{P K C} c_{\text {mutant }}=7.2 \mathrm{~s} ; \mathrm{PKC} \gamma: \mathrm{DGK} \gamma: 1: 0.5, \tau_{\mathrm{PKC}} \mathrm{m}_{\text {mutant }}=9.1 \mathrm{~s} ; \mathrm{PKC} \gamma: \mathrm{DGK} \gamma: 1: 0.3, \tau_{\mathrm{PKC}} \gamma$ mutant $\left.=12.1 \mathrm{~s}\right)$. (B) Translocation characteristics of PKC $\gamma$ in the wild-type model. Here, results indicate that reducing the PKC $\gamma$ :DGK $\gamma$ expression ratio first decreases and then increases the membrane residence time of $\gamma$ isoform (PKC $\gamma: D G K \gamma: 1: 1, \tau_{P_{K C}}=18 \mathrm{~s} ;$ PKC $\gamma: D G K \gamma: 1: 0.5, \tau_{P K C}$ mutant $=17 \mathrm{~s} ;$ PKC $\gamma: D G K \gamma: 1: 0.3, \tau_{P K C}$ mutant $\left.=19.8 \mathrm{~s}\right)$.

that constitutively active PKC $\gamma$ may have shorter membrane residence duration in a mutant model of cPCs, despite higher activity levels (Adachi et al., 2008; Shuvaev et al., 2011; Wong et al., 2018).

In this study, we explore the mechanisms that might control membrane residence time of PKC $\gamma$. Our results suggest that the formation of a local signalosome in the membrane compartment is key in regulating the membrane residence time of $\mathrm{PKC} \gamma$ (Yamaguchi et al., 2006). Furthermore, our results show that, in the case of the wild-type model, the local signalosome is formed in the membrane compartment. In the case of the mutant model, the signalosome is formed in the cytosol. It seems that in the wild-type model, the depolarization-induced stimulation leads to membrane translocation of inactive PKC $\gamma$ and DGK $\gamma$. Once in the membrane compartment, these molecules organize themselves into a local signalosome, which, in turn, leads to DAG metabolism and eventual disassembly of this local signaling machine. It also leads to return of both DAG effector molecules to the cytosol. Our results suggest that, in the mutant model, the signalosome between PKC $\gamma$ and DGK $\gamma$ is formed in the cytosol. This occurs independent of DAG and even under basal conditions.

Our results propose that, in the case of the wild-type model, the molecular processes involved in the assembly, anchoring, and disassembly of the signalosome may provide enough time for $\mathrm{PKC} \gamma$ to tightly modulate the activation, inactivation, or recruitment of its key substrates, TRPC3 channels in PCs. In contrast, our simulations suggest that, in the mutant model, signalosome is not assembled at the membrane. Instead, the PKC $\gamma$ and DGK $\gamma$ molecular pair interact with each other in the cytosol under basal and DAG-independent conditions. Furthermore, the results reflect that reduced residence time of $\mathrm{PKC} \gamma$ in the mutant model could be due to the lack of signalosome assembly at the membrane. This is because depolarization-induced translocation of active DGK $\gamma$ molecule from the cytosol to the membrane could lead to direct metabolism of DAG into PA, without undergoing the formation of a molecular complex at the membrane. Consequently, this results in faster DAG conversion to $\mathrm{PA}$ and quicker return of effector molecules 
to the cytosol. These results support the idea that reduced membrane residence time of PKC $\gamma$ is mainly linked to its constitutive activity in the cytosol (Wong et al., 2018), which, in turn, may result in differences in $\mathrm{PKC} \gamma$ and $\mathrm{DGK} \gamma$ interaction in the cytosol.

A critical assumption of this study is based on the possibility of a functional coupling between DAG effector molecules in PCs. This assumption could be disputed, as we do not have a direct evidence that such a coupling exists in PCs and is functional during the depolarization-induced translocation and activation events. We borrowed the idea of this functional interaction between DAG effector molecules from a previous experimental observation focusing on the PKC $\gamma$ and DGK $\gamma$ interactions in $\mathrm{CHO}$ cells and our previous modeling work of this system (Yamaguchi et al., 2006; Aslam and Alvi, 2019). We assumed that a similar signaling cascade could be functional in PCs. This similarity of CHO model cell and PCs could be disputed, as there may be differences in translocation dynamics of DGK $\gamma$ in these cell systems. However, another set of direct observations in PCs indicate the quick degradation of DAG, cyctosol-tomembrane translocation kinetics of $\mathrm{PKC} \gamma$, remigration dynamics of $\mathrm{PKC} \gamma$ to cytosol after purinergic receptor activation, and membrane residence duration in both mutant and wild-type models (Shuvaev et al., 2011). Based on these observations, we argue that during membrane depolarization-induced events in PCs, a coupling between DAG effector molecules could be present and functional. In the absence of a net negative feedback effect generated through PKC $\gamma$ and DGK $\gamma$ interactions, DAG might persist at membrane, leading to the prolonged membrane residence duration of $\mathrm{PKC} \gamma$, which at least is not the case, as observed in some previous studies (Adachi et al., 2008; Shuvaev et al., 2011; Wong et al., 2018). In fact, the mutant PKC $\gamma$ molecule is linked to shorter membrane residence duration (Adachi et al., 2008; Shuvaev et al., 2011). Additional observations in the SCA14 mutant model (Wong et al., 2018) suggest the mislocalization of PKC $\gamma$ in cytosol with hyperactivity and the possibility of phosphorylating/activating its substrates in the cytosol.

Besides exploring depolarization-induced translocation characteristics of $\mathrm{PKC} \gamma$ and $\mathrm{DGK} \gamma$ molecules in mutant and wild-type models of PCs, we have also studied the influence of stimulation strength (amplitude of pulse mimicking depolarization event) on the translocation characteristics and membrane residence time of $\mathrm{PKC \gamma}$ (Figures $4 \mathbf{A}, \mathbf{B}$ ). Our results indicate that, in mutant models, the maximum $\mathrm{M} / \mathrm{C}$ ratio of $\mathrm{PKC} \gamma$ increases fourfold with a threefold increase in stimulation strength. In contrast, the increase is 10 -fold for the same increase in stimulation strength for models representing wild-type PCs. Interestingly, the membrane residence time of $\mathrm{PKC} \gamma$ in the mutant model first decreases and then increases with increasing stimulation strength. The corresponding residence and translocation characteristics of DGK $\gamma$ shows decreases in membrane residence but increases in the maximum $\mathrm{M} / \mathrm{C}$ ratio for this molecule with increasing stimulation strength (Supplementary Figure S1).

Furthermore, our results show that a 25 - to 100 -fold increase in the DAG metabolism rate leads to a 1-s reduction in membrane residence duration for the mutant and a 2$s$ reduction for the wild-type model. The maximum M/C ratio is reduced $10 \%$ in the mutant and $40 \%$ in the wildtype model (Figure 5). This occurs because enhancing DAG metabolism results in decreased membrane translocation and faster remigration to the cytosol. Interestingly, blocking second messenger metabolism has an opposite effect on membrane residence duration, as well as the translocation characteristics of PKC $\gamma$ (Figure 6). This occurs because blocking metabolism will result in DAG accumulation at the membrane compartment, thus enhancing the membrane residence duration, as well as the $\mathrm{M} / \mathrm{C}$ ratio of $\mathrm{PKC} \gamma$. Interestingly, our results show that the effects of metabolism blocking are much more pronounced on membrane residence duration in mutant PKC $\gamma$ (95\% blocking of DAG metabolism results in a twofold increase in residence time) compared to the wild-type model (95\% blocking results only in a $10 \%$ increase in residence time) (Figure 6). However, in the case of a maximum $\mathrm{M} / \mathrm{C}$ ratio, 95\% blocking of second messenger metabolism in the wild-type model results in a 5-fold increase. This increase is only 2 -fold in the mutant model (Figure 6). The corresponding residence and translocation characteristics of DGK $\gamma$ shows increase in the maximum $\mathrm{M} / \mathrm{C}$ ratio for this molecule in the wild-type model with increase in blocking of DAG metabolism rate (Supplementary Figure S2).

Our results also indicate a role for DGK $\gamma$ expression on the membrane residence duration and translocation characteristics of PKC $\gamma$ (Figure 8). Recent experimental results that use the GFP-tagged PKC $\gamma$ in $\mathrm{CHKO}$ cells indicate that the membrane residence of $\mathrm{PKC} \gamma$ could be modulated by negative feedback effects of $\mathrm{DGK} \gamma$ on the activity of $\mathrm{PKC} \gamma$ through regulating DAG metabolism (Yamaguchi et al., 2006). However, these results are based on a wild-type system. It is not understood how this negative feedback loop is affected in mutant systems when PKC $\gamma$ is constitutively active. In addition, how might this influence the membrane residence time, as well as the $\mathrm{M} / \mathrm{C}$ ratio of mutant PKC $\gamma$ ? These models assume equally proportional DGK $\gamma$ expression is necessary for its negative influence on PKC $\gamma$ activity. The results indicate that any changes in expression ratios of PKC $\gamma$ and DGK $\gamma$ will have a strong influence on the membrane residence time and $\mathrm{M} / \mathrm{C}$ ratio of $\mathrm{PKC} \gamma$ and DGK $\gamma$ (Figure 8 and Supplementary Figure S3). These results are consistent with previous observations (Yamaguchi et al., 2006) showing a fourfold increase in membrane residence time of PKC $\gamma$. However, the key difference between the kinetics reported in previous experimental findings and the results reported here is that the current results are based on depolarization-induced stimulation in PCs (Shuvaev et al., 2011) and experimental observations mentioned above are based on ATP-induced stimulation in $\mathrm{CHO}$ cells (Yamaguchi et al., 2006).

In our models, we did not attempt to simulate cytosol-tomembrane translocation and re-translocation back to the cytosol in a detailed manner (Imai et al., 2002, 2004; Luo et al., 2003a,b). This study only represented the whole complex process by introducing two first-order functions of DAG concentration. These functions describe the cytosol-to-membrane migration rates of PKC $\gamma$ and DGK $\gamma$ as a linear function of DAG 
concentration (Supplementary Material S1: Table S1 and Supplementary Material S3: Table S2). The slope of the PKC $\gamma$ function is higher compared to DGK $\gamma$ and adjusted to account for differential sensitivity of these molecules to DAG and calcium concentrations (Yamaguchi et al., 2006). We are not aware if there is any model proposed to describe the $\mathrm{PKC} \gamma$ and DGK $\gamma$ translocation in PCs. Clearly, a complete model should account for events like DAG and $\mathrm{IP}_{3}$ generation, DAG binding, DAG-induced activation of TRPC3, and $\mathrm{Ca}^{+2}$ influx due to TRPC3 opening. In addition, there is $\mathrm{IP}_{3}$ diffusion into cytosol, $\mathrm{Ca}^{+2}$ diffusion from membrane to cytosol, $\mathrm{Ca}^{+2}$ release from intracellular stores, binding of $\mathrm{Ca}^{+2}$ with dormant PKC $\gamma$, and translocation of PKC $\gamma$ and DGK $\gamma$ from the cytosol to the membrane (Nishizuka, 1988, 1992, 1995; Newton, 2001, 2003; Oancea and Meyer, 1998). In addition, diffusion in the membrane occurs. However, this is beyond the scope of this study. Similarly, the models presented in this study assume that the complex process of DAG generation in response to depolarization-induced activation of the mGluR1 pathway can be simply represented by a brief pulse with certain duration and amplitude. This study has not modeled the complex processes involved in the generation of DAG and $\mathrm{IP}_{3}$ after the activation of the G-protein-coupled phospholipase. Rather, we have used a simplistic approach to describe these complex processes.

Some of the assumptions we made to construct these models may be disputed. This study was not able to find data points linking the effect of PKC $\gamma$ mutations to the extent of its activity. Do these mutations lead to maximal activation or only partial activation of $\mathrm{PKC} \gamma$ in the cytosol? Here, we assume that mutations in $\mathrm{PKC} \gamma$ lead to maximum basal activation, which, in turn, leads to the maximum phosphorylation and activation of DGK $\gamma$ in the cytosol, even during resting conditions. According to our mutant model, depolarization-induced local generation of DAG in the membrane compartment stimulates the translocation of its already-active effector molecules from the cytosol to the membrane. Active and phosphorylated DGK $\gamma$ is in the membrane compartment and stimulates the DAG metabolism. This assumption may be disputed, and additional data should be generated to evaluate the DAG-binding capacity of constitutively active mutant molecule. Here, this study assumes that DAG metabolism in the mutant model is fast, and as soon DAG levels drop, both molecules relocate back to the cytosol.

Based on our wild-type and mutant models, it appears that activation and phosphorylation of DGK $\gamma$ could critically influence the magnitude of translocation as well as the membrane residence duration of $\mathrm{PKC} \gamma$ in both these models (Figure 7). The blocking of DGK $\gamma$ activation in the mutant model clearly shows that the magnitude of translocation and the membrane residence time of PKC $\gamma$ are increased (Figure 7A). However, the same blocking in the wild-type model shows that though the translocation intensity increases, membrane residence time decreases with increase in blocking levels (Figure 7B). This is rather counterintuitive, as one would expect the increase in membrane residence duration with blocking of DGK $\gamma$ activation in wild-type models too. Interestingly, as the parameter $\mathrm{k}_{6}$ was further blocked, we noticed that 99 and $99.95 \%$ blocking resulted in an increase not only in the magnitude of translocation but also in membrane residence duration (Supplementary Figure S4). Thus, these results indicate that reducing the activation of $\mathrm{DGK} \gamma$ reduces the efficacy of negative feedback loop.

\section{CONCLUSION}

Through a computational approach, we show that enhanced PKC $\gamma$ activity is linked to reduced membrane residence duration in the SCA14 mutant model. This work provides the very first simple mechanistic understanding and comparison of PKC $\gamma$ temporal dynamics in wild-type and mutant models. We propose that SCA14 mutation causes the shift of PKC $\gamma$ signaling from membrane compartment to cytosol, thus resulting in reduced membrane lifetime. The mechanistic nature of this work provides possibilities to increase the membrane residence duration of PKC $\gamma$ through specific interventions, exquisitely targeting biochemical interactions such as blocking the DAG metabolism rate. An integrated model describing the calcium homeostasis in PCs involving DAG effector molecules and TRPC3 channels is under development and could further enhance the insights of DAG signaling in PCs.

\section{DISCLOSURE}

The corresponding author is part of a non-profit, i.e., BioSystOimcs, focusing on the basic R\&D in drug target identification and development with no commercial plans and interests.

\section{DATA AVAILABILITY STATEMENT}

The datasets generated for this study are available on request to the corresponding author.

\section{AUTHOR CONTRIBUTIONS}

NA came up with the idea, carried out modeling and wrote the manuscript. FA reviewed data and helped in polishing ideas.

\section{ACKNOWLEDGMENTS}

Special thanks to Mrs. Bushria Aslam for support and inspiration.

\section{SUPPLEMENTARY MATERIALS}

The Supplementary Material for this article can be found online at: https://www.frontiersin.org/articles/10.3389/fnins.2019. 01397/full\#supplementary-material 


\section{REFERENCES}

Adachi, N., Kobayashi, T., Takahashi, H., Kawasaki, T., Shirai, Y., Ueyama, T., et al. (2008). Enzymological analysis of mutant protein kinase $\mathrm{C} \gamma$ causing spinocerebellar ataxia type 14 and dysfunction in Ca2+ homeostasis. J. Biol. Chem. 283, 19854-19863. doi: $10.1074 /$ jbc.m801492200

Aslam, N., and Alvi, F. (2017). How the cytosol-to-membrane translocation kinetics and signaling of $\mathrm{PKC} \gamma$ are dysregulated in the neurodegenerative spinocerebellar ataxia type14 (SCA14). FASEB J. 31:183.

Aslam, N., and Alvi, F. (2019). How the PKC $\gamma$ activity is regulated in the neurodegenerative spinocerebellar ataxia type 14 (SCA14)? FASEB J. 30:1179.

Brkanac, Z., Bylenok, L., Fernandez, M., Matsushita, M., Lipe, H., Wolff, J., et al. (2002). A new dominant spinocerebellar ataxia linked to chromosome 19q13.4-qter. Arch. Neurol. 59, 1291-1295.

Carlson, K. M., Andresen, J., and Orr, H. T. (2009). Emerging pathogenic pathways in the spinocerebellar ataxias. Curr. Opin. Genet. Dev. 19, 247-253. doi: 10.1016/ j.gde.2009.02.009

Chen, C., Kano, M., Abeliovich, A., Chen, L., Bao, S., Kim, J. J., et al. (1995). Impaired motor coordination correlates with persistent multiple climbing fiber innervation in PKC gamma mutant mice. Cell 29, 1233-1242. doi: 10.1016/ 0092-8674(95)90148-5

Duenas, A. M., Goold, R., and Giunti, P. (2006). Molecular pathogenesis of spinocerebellar ataxias. Brain 129, 1357-1370. doi: 10.1093/brain/awl081

Goto, K., and Kondo, H. (1999). Diacylglycerol kinase in the central nervous system molecular heterogeneity and gene expression. Chem. Phys. Lipids 98, 109-117. doi: 10.1016/s0009-3084(99)00023-7

Guzman, S. J., and Gerevich, Z. (2016). P2Y receptors in synaptic transmission and plasticity: therapeutic potential in cognitive dysfunction. Neural Plast. 2016:1207393. doi: 10.1155/2016/1207393

Henning, H. A. (2011). Synaptic Signaling by mGluR1 and TRPC3 in Spiny Dendrites of Cerebellar Purkinje Cells. Ph.D. Dissertation, Technische Universitat Munchen, Munchen.

Imai, S., Kai, M., Yamada, K., Kanoh, H., and Sakane, F. (2004). The plasma membrane translocation of diacylglycerol kinase $\delta 1$ is negatively regulated by conventional protein kinase C-dependent phosphorylation at Ser-22 and Ser26 within the pleckstrin homology domain. Biochem. J. 382, 957-966. doi: $10.1042 /$ bj20040681

Imai, S., Sakane, F., and Kanoh, H. (2002). Phorbol ester-regulated oligomerization of diacylglycerol kinase $\delta$ linked to its phosphorylation and translocation. J. Biol Chem. 278, 39542-39547.

Kano, M., Hashimoto, K., Chen, C., Abeliovich, A., Aiba, A., Kurihara, H., et al. (1995). Impaired synapse elimination during cerebellar development in PKC gamma mutant mice. Cell 83, 1223-1231. doi: 10.1016/0092-8674(95)90147-7

Luo, B., Prescott, S. M., and Topham, M. K. (2003a). Association of diacylglycerol kinase $\zeta$ with protein kinase $\mathrm{C} \alpha$ spatial regulation of diacylglycerol signaling. J. Cell Biol. 160, 929-937.

Luo, B., Prescott, S. M., and Topham, M. K. (2003b). Protein Kinase C $\alpha$ Phosphorylates and Negatively Regulates Diacylglycerol Kinase $\zeta$. J. Biol. Chem. 278, 39542-39547. doi: 10.1074/jbc.m307153200

Mérida, I., Avila-Flores, A., and Merino, E. (2008). Diacylglycerol kinases: at the hub of cell signaling. Biochem. J. 409, 1-18. doi: 10.1042/bj20071040

Metzger, F., and Kapfhammer, J. P. (2000). Protein kinase C activity modulates dendritic differentiation of rat Purkinje cells in cerebellar slice cultures. Eur. J. Neurosci 12, 1993-2005. doi: 10.1046/j.1460-9568.2000.00086.x

Newton, A. C. (2001). Protein kinase C: structural and spatial regulation by phosphorylation, cofactors, and macromolecular interactions. Chem. Rev. 101, 2353-2364. doi: $10.1021 / \mathrm{cr} 0002801$

Newton, A. C. (2003). Regulation of the ABC kinases by phosphorylation: protein kinase $\mathrm{C}$ as a paradigm. Biochem. J. 370, 361-371. doi: 10.1042/bj20021626

Nishizuka, Y. (1988). The molecular heterogeneity of protein kinase C and its implications for cellular regulation. Nature 334, 661-665. doi: 10.1038/ $334661 \mathrm{a} 0$

Nishizuka, Y. (1992). Intracellular signaling by hydrolysis of phospholipids and activation of protein kinase C. Science 258, 607-614. doi: 10.1126/science. 1411571
Nishizuka, Y. (1995). Protein kinase C and lipid signaling for sustained cellular responses. FASEB J. 9, 484-496. doi: 10.1096/fasebj.9.7.7737456

Oancea, E., and Meyer, T. (1998). Protein kinase C as a molecular machine for decoding calcium and diacylglycerol signals. Cell 95, 307-318. doi: 10.1016/ s0092-8674(00)81763-8

Paulson, H. L. (2009). The spinocerebellar ataxias. J. Neuroophthalmol. 29, 227237. doi: 10.1097/WNO0b013e3181b416de

Saito, N., Kikkawa, U., Nishizuka, Y., and Tanaka, C. (1988). Distribution of protein kinase C like immunoreactive neurons in rat brain. J. Neurosci. 8, 369-382. doi: 10.1523/jneurosci.08-02-00369.1988

Saito, N., and Shirai, Y. (2002). Protein kinase $\mathrm{C} \gamma(\mathrm{PKC} \gamma)$ : function of neuron specific isotype. J. Biochem. 132, 683-687. doi: 10.1093/oxfordjournals.jbchem. a003274

Schrenk, K., Kapfhammer, J. P., and Metzger, F. (2002). Altered dendritic development of cerebellar Purkinje cells in slice cultures from protein kinase Cgamma-deficient mice. Neuroscience 110, 675-689. doi: 10.1016/s03064522(01)00559-0

Shimobayashi, E. (2016). Mechanisms of PKC Gamma-Mediated Inhibition of Dendritic Growth in Cerebellar Purkinje Cells. Ph.D. Dissertation, Universität Basel, Basel.

Shuvaev, A. N., Horiuchi, H., Seki, T., Goenawan, H., Irie, T. I., Iizuka, A., et al. (2011). Mutant PKC $\gamma$ in spinocerebellar ataxia type 14 disrupts synapse elimination and long-term depression in purkinje cells In Vivo. J. Neurosci. 30, 14324-14334. doi: 10.1523/jneurosci.5530-10.2011

Soong, B. W., and Paulson, H. L. (2007). Spinocerebellar ataxias: an update. Curr. Opin. Neurol. 20, 438-446. doi: 10.1097/wco.0b013e3281fbd3dd

Verbeek, D. S., Goedhart, J., Bruinsma, L., Sinke, R. J., and Reits, E. A. (2008). PKC gamma mutations in spinocerebellar ataxia type 14 affect $\mathrm{C} 1$ domain accessibility and kinase activity leading to aberrant MAPK signaling. J. Cell Sci. 121(Pt 14), 2339-2349. doi: 10.1242/jcs.027698

Verbeek, D. S., Knight, M. A., Harmison, G. G., Fischbeck, K. H., and Howell, B. W. (2005). Protein kinase C gamma mutations in spinocerebellar ataxia 14 increase kinase activity and alter membrane targeting. Brain 128, 436-442. doi: 10.1093/brain/awh378

Weisman, G. A., Camden, J. M., Peterson, T. S., Ajit, D. V., Woods, L. T., and Erb, L. (2012). Receptors for extracellular nucleotides in the central nervous system: role of P2X7 and P2Y2 receptor interactions in neuroinflammation. Mol. Neurobiol. 46, 96-113. doi: 10.1007/s12035-012-8263-z

Wong, M. M. K., Hoekstra, S. D., Vowles, J., Waston, L. M., Fuller, G., Nemeth, A. H., et al. (2018). Neurodegeneration in SCA14 is associated with increased PKC $\gamma$ kinase activity, mislocalization and aggregation. Acta Neuropathol. Commun. 6:99.

Yabe, I., Sasaki, H., Chen, D. H., Raskind, W. H., Bird, T. D., Yamashita, I., et al. (2003). Spinocerebellar ataxia type 14 caused by a mutation in protein kinase $\mathrm{C}$ gamma. Arch. Neurol. 60, 1749-1751.

Yamaguchi, Y., Shirai, Y., Matsubara, T., Sanse, K., Kuriyama, M., Oshiro, N., et al. (2006). Phosphorylation and Up-regulation of diacylglycerol kinase $\gamma$ via its interaction with protein kinase C $\gamma$. J. Biol. Chem. 281, 31627-31637. doi: 10.1074/jbc.m606992200

Yamashita, I., Sasaki, H., Yabe, I., Fukazawa, T., Nogoshi, S., Komeichi, K., et al. (2000). A novel locus for dominant cerebellar ataxia (SCA14) maps to a 10.2-cM interval flanked by D19S206 and D19S605 on chromosome 19q13.4- qter. Ann. Neurol. 48, 156-163. doi: 10.1002/1531-8249(200008)48:2<156::aid-ana4>3.0. co;2-9

Conflict of Interest: The authors declare that the research was conducted in the absence of any commercial or financial relationships that could be construed as a potential conflict of interest.

Copyright $\odot 2020$ Aslam and Alvi. This is an open-access article distributed under the terms of the Creative Commons Attribution License (CC BY). The use, distribution or reproduction in other forums is permitted, provided the original author(s) and the copyright owner(s) are credited and that the original publication in this journal is cited, in accordance with accepted academic practice. No use, distribution or reproduction is permitted which does not comply with these terms. 